\title{
Dynamic Analysis of a High-Static-Low-Dynamic-Stiffness Vibration Isolator with Time-Delayed Feedback Control
}

\author{
Yong Wang, Shunming Li, Chun Cheng, and Xingxing Jiang \\ College of Energy and Power Engineering, Nanjing University of Aeronautics and Astronautics, Nanjing 210016, China \\ Correspondence should be addressed to Yong Wang; wangy1921@126.com
}

Received 11 November 2014; Revised 1 March 2015; Accepted 5 March 2015

Academic Editor: Lei Zuo

Copyright ( 2015 Yong Wang et al. This is an open access article distributed under the Creative Commons Attribution License, which permits unrestricted use, distribution, and reproduction in any medium, provided the original work is properly cited.

\begin{abstract}
This paper proposes the time-delayed cubic velocity feedback control strategy to improve the isolation performance of High-StaticLow-Dynamic-Stiffness (HSLDS) vibration isolator. Firstly, the primary resonance of the controlled HSLDS vibration isolator is obtained by using multiple scales method. The equivalent damping ratio and equivalent resonance frequency are defined to study the effects of feedback gain and time delay on the primary resonance. The jump phenomenon analysis of the controlled system without and with time delay is investigated by using Sylvester resultant method and optimization method, respectively. The stability analysis of the controlled system is also considered. Then, the $1 / 3$ subharmonic resonance of the controlled system is studied by using multiple scales method. The effects of feedback gain and time delay on the $1 / 3$ subharmonic resonance are also presented. Finally, force transmissibility is proposed to evaluate the performance of the controlled system and compared with an equivalent linear passive vibration isolator. The results show that the vibration amplitude of the controlled system around the resonance frequency region decreases and the isolation frequency band is larger compared to the equivalent one. A better isolation performance in the high frequency band can be achieved compared to the passive HSLDS vibration isolator.
\end{abstract}

\section{Introduction}

Recently passive nonlinear vibration isolators with HighStatic-Low-Dynamic-Stiffness (HSLDS) characteristic $[1,2]$ have drawn much attention by many researchers, since they can have a high-static stiffness with a small static displacement without sacrificing the load bearing capacity and a small dynamic stiffness to achieve a low natural frequency which can perform better than the linear ones. The HSLDS vibration isolator is comprised of a load bearing elastic element providing positive stiffness and special mechanisms providing negative stiffness called stiffness correctors. The load bearing elastic element is usually the vertical spring and the stiffness correctors have various types. Carrella, Kovacic, and Wang et al. [3-6] used inclined springs as stiffness correctors to build a HSLDS vibration isolator and studied the static and dynamic behaviors theoretically. Le and Ahn [7] considered a HSLDS vibration isolator composed of two symmetric negative stiffness structures and a positive stiffness structure for improving vibration isolation performance of the vehicle seat. Robertson, Zhou, Xu, and Shin et al. [8-11] built the HSLDS vibration isolators using electromagnetic springs or magnetic springs as stiffness correctors and analyzed the static and dynamic characteristics theoretically and experimentally. Liu et al. $[12,13]$ built a HSLDS vibration isolator by using Euler buckled beams as stiffness correctors and investigated the dynamic characteristics systematically. Shaw et al. $[14,15]$ used bistable composite plate as stiffness correctors to build a HSLDS vibration isolator and analyzed the steady state response detailedly. After being loaded at the static equilibrium position, the negative stiffness provided by the stiffness correctors is exactly balanced by the positive stiffness of the vertical spring; then a small dynamic stiffness can be obtained to achieve a low natural frequency.

The dynamic motion of the HSLDS vibration isolator under force or base excitation can be approximately described by the Duffing equation with linear and cubic stiffness terms. Because of the inherent nonlinearity in the HSLDS vibration isolator, with the increase of the excitation force or base amplitude, the resonance peak can become large and jump phenomenon can occur at the resonant frequency which are undesirable conditions in practical engineering. Therefore, the HSLDS 
vibration isolator should be improved to obtain better isolation performance. Increasing the linear damping can reduce the vibration amplitude around the resonance frequency band, avoid the jump phenomenon, and obtain a larger isolation frequency band but results in a poor performance in the high frequency band where the isolation is required. Passive vibration isolators often meet the dilemma between these characteristics; this can be eliminated by using active control strategy. The active control of the Duffing oscillator has been studied by many researchers [16-20]; because of the unavoidable time delay in controllers and actuators, the effects of time delay are considered in the active control strategy. The active control strategy is usually the time-delayed linear velocity or time-delayed linear displacement control strategy. Few studies have drawn attention to the active control of the HSLDS vibration isolator; Sun et al. [21] used the time-delayed linear displacement control strategy to obtain better isolation performance around the resonance frequency band under both force and base excitation. Although these mentioned strategies can be used to achieve better isolation performance of the HSLDS vibration isolator around the resonance frequency band and obtain a larger isolation frequency band under both force and base excitation, the dilemma as the passive vibration isolator case can exist in these active control strategies. So in order to overcome this dilemma, this paper proposes the time-delayed cubic velocity feedback control strategy. Compared to the above-mentioned strategies, the time-delayed cubic velocity feedback control strategy can not only obtain better isolation performance around the resonance frequency band and obtain a larger isolation frequency, but also achieve a better performance in the high frequency band where the isolation is required.

The organization of this paper is as follows. A HSLDS vibration isolator with time-delayed cubic velocity feedback control is presented and brief description of static analysis is shown in Section 2. In Section 3, the primary resonance of the controlled HSLDS vibration isolator is obtained by using the multiple scales method. The jump phenomenon analysis of the controlled system without and with time delay is investigated by using the Sylvester resultant method and optimization method, respectively. The stability analysis of the controlled system is then studied. The effects of the feedback gain and time delay on the primary resonance, jump phenomenon, and stability are also considered. In Section 4, the $1 / 3$ subharmonic resonance is studied by using the multiple scales method. The effects of the feedback gain and time delay on the existence regions where $1 / 3$ subharmonic resonance occurs are also presented. In Section 5, the force transmissibility of the controlled HSLDS vibration isolator is obtained and compared with an equivalent linear passive vibration isolator. Conclusions are drawn in Section 6.

\section{Modeling of a HSLDS Vibration Isolator}

A HSLDS vibration isolator with time-delayed cubic velocity feedback control is shown in Figure 1. The HSLDS vibration isolator is comprised of vertical spring used as load bearing element and two symmetric stiffness correctors providing negative stiffness.

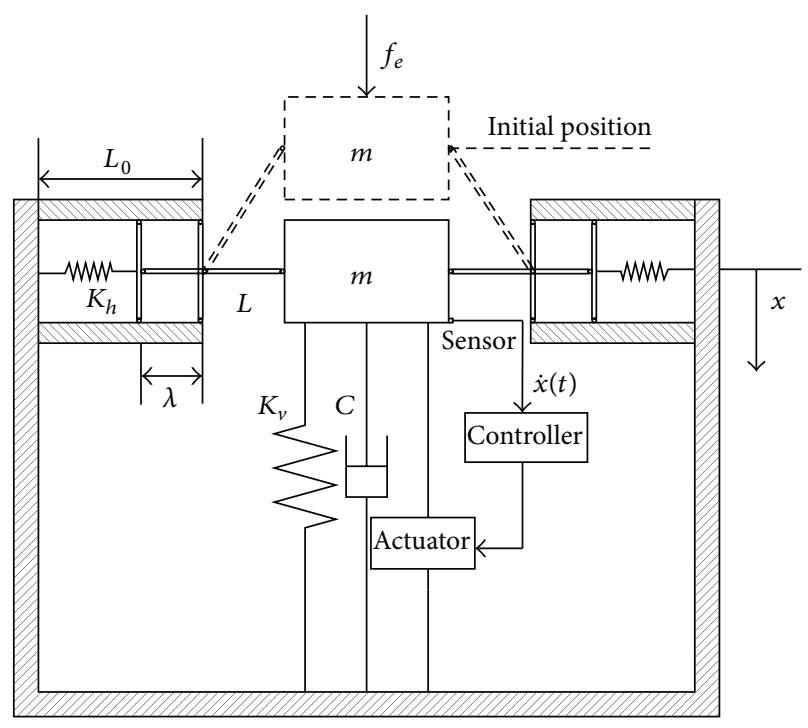

FIGURE 1: Scheme of a HSLDS vibration isolator with time-delayed cubic velocity feedback control.

Figure 1 also shows when loading a mass $m$ the system is balanced at the static equilibrium position. The stiffness of the vertical and horizontal springs is $K_{v}$ and $K_{h}$; the initial length of the horizontal springs is $L_{0}$; the compression deformation of the horizontal springs when the system is at the static equilibrium position is $\lambda$; the length of the connecting element is $L ; x$ is the displacement of the mass from the static equilibrium position; the damping coefficient of the damping is $C$; and the excitation is force excitation with a harmonic force $f_{e}=F_{e} \cos (w t)$. The velocity of the mass is measured by the sensor; the velocity signal $\dot{x}$ can be exported to a certain cubic velocity feedback signal through the nonlinear controller and actuator. Because of the unavoidable time delay in the controller and actuator, the effects of time delay should be considered; then the time-delayed cubic velocity feedback control strategy can be implemented.

The force-displacement and stiffness-displacement relationships of the system are given as

$$
\begin{aligned}
& F=K_{v} x-2 K_{h}\left[\lambda-\left(L-\sqrt{L^{2}-x^{2}}\right)\right] \frac{x}{\sqrt{L^{2}-x^{2}}}, \\
& K=\left(K_{v}-2 K_{h}\right)+\frac{2 K_{h}(L-\lambda) L^{2}}{\left(L^{2}-x^{2}\right)^{3 / 2}} .
\end{aligned}
$$

Equations (1a) and (1b) can be written in nondimensional form as

$$
\begin{aligned}
\widehat{F} & =(1-2 k) \widehat{x}+2 k(1-\widehat{\lambda}) \frac{\widehat{x}}{\sqrt{1-\widehat{x}^{2}}}, \\
\widehat{K} & =(1-2 k)+\frac{2 k(1-\widehat{\lambda})}{\left(1-\widehat{x}^{2}\right)^{3 / 2}},
\end{aligned}
$$

where $\widehat{x}=x / L, \hat{\lambda}=\lambda / L, k=K_{h} / K_{v}, \widehat{F}=F /\left(K_{v} L\right)$, and $\widehat{K}=K / K_{v}$. 

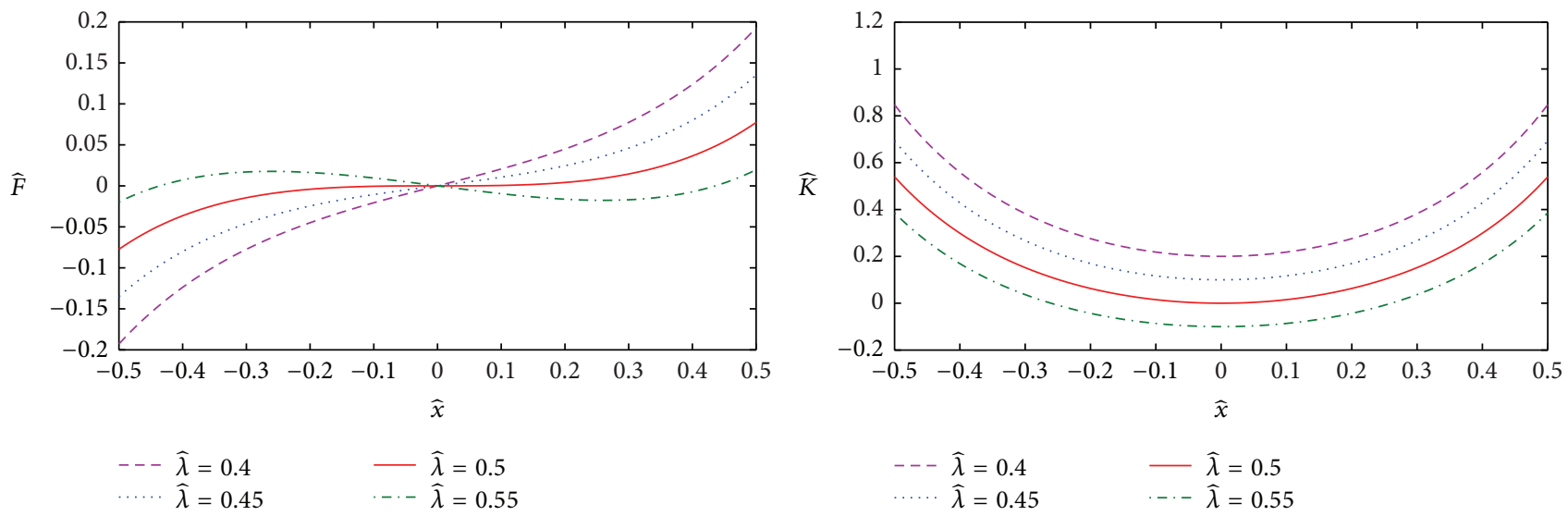

FIGURE 2: Nondimensional force-displacement and stiffness-displacement curves.

The nondimensional stiffness at the static equilibrium position can be obtained by substituting $\widehat{x}=0$ into ( $2 \mathrm{~b}$ ):

$$
\widehat{K}_{s}=1-2 k \widehat{\lambda} .
$$

If the stiffness of the HSLDS vibration isolator is zero at the static equilibrium position, the quasi-zero-stiffness (QZS) characteristic can be obtained; then the value of $\hat{\lambda}$ is given as

$$
\widehat{\lambda}_{\mathrm{qzs}}=\frac{1}{2 k} \text {. }
$$

The nondimensional force-displacement and stiffnessdisplacement curves of the HSLDS vibration isolator for various values of $\widehat{\lambda}$ when $k=1$ are shown in Figure 2. It can be clearly seen that if $\hat{\lambda}=\widehat{\lambda}_{\text {qzs }}$, the negative stiffness provided by the stiffness correctors is exactly balanced by the positive stiffness of the vertical spring in the vertical direction at the static equilibrium position; then the QZS characteristic can be achieved. If $\hat{\lambda}>\widehat{\lambda}_{\mathrm{qz}}$, the stiffness is negative in the neighborhood of the static equilibrium position; then the system can be unstable which is an undesirable condition in practical engineering. Because of some inevitable errors in practical conditions, the QZS characteristic can be hard to achieve and in order to ensure the system stability, the stiffness of the system should keep a small positive value at the static equilibrium position, so the system can have a common HSLDS characteristic. In this paper, the case $\hat{\lambda}<\hat{\lambda}_{\text {qzs }}$ is considered.

When the amplitude of the displacement is small, the nondimensional force and stiffness can be expanded as a Taylor series at the static equilibrium position $\widehat{x}=0$ for simplicity

$$
\begin{aligned}
\widehat{F}_{a}(\widehat{x})=(1-2 k \widehat{\lambda}) \widehat{x} & +k(1-\widehat{\lambda}) \widehat{x}^{3}=\alpha \widehat{x}+\gamma \widehat{x}^{3}, \\
\widehat{K}_{a}(\widehat{x}) & =\alpha+3 \gamma \widehat{x}^{2}, \\
\alpha & =1-2 k \widehat{\lambda}, \\
\gamma & =k(1-\widehat{\lambda}) .
\end{aligned}
$$

\section{Primary Resonance}

3.1. Steady State Resonance. The dynamic equation of the HSLDS vibration isolator with time-delayed cubic velocity feedback control under harmonic force excitation using third-order Taylor series expansion is given as

$$
m \ddot{x}+C \dot{x}+C_{3} \dot{x}^{3}(t-\delta)+K_{v} \alpha x+\gamma K_{v} \frac{x^{3}}{L^{2}}=F_{e} \cos w t,
$$

where $C_{3}$ and $\delta$ are the feedback gain and time delay, respectively; the dots denote derivatives with respect to time $t$. Equation (6) can be written in nondimensional form as

$$
\widehat{x}^{\prime \prime}+2 \zeta_{1} \widehat{x}^{\prime}+2 \zeta_{3} \widehat{x}^{\prime 3}(T-\tau)+\widehat{x}+\beta \widehat{x}^{3}=f_{1} \cos \Omega T,
$$

where $\hat{x}=x / L, \zeta_{1}=C /\left(2 m w_{n}\right), \zeta_{3}=C_{3} L^{2} w_{n} /(2 m), \beta=\gamma / \alpha$, $w_{n}=\sqrt{\alpha K_{v} / m}, \Omega=w / w_{n}, T=w_{n} t, f_{1}=F_{e} /\left(\alpha K_{v} L\right)$, and $\tau=w_{n} \delta$. The primes denote derivatives with respect to $T$. To analyze the primary resonance of the controlled system under force excitation by using the multiple scales method, it is assumed that

$$
\begin{gathered}
\zeta_{1}=\varepsilon \widehat{\zeta}_{1}, \\
\zeta_{3}=\varepsilon \widehat{\zeta}_{3}, \\
\beta=\varepsilon \widehat{\beta}, \\
f_{1}=\varepsilon f, \\
\Omega^{2}=1+\varepsilon \sigma,
\end{gathered}
$$

where $\varepsilon$ is a formal small parameter and $\sigma$ is the detuning frequency. Then it is convenient to rewrite (7) as

$$
\begin{aligned}
\widehat{x}^{\prime \prime}+ & 2 \varepsilon \widehat{\zeta}_{1} \widehat{x}^{\prime}+2 \varepsilon \widehat{\zeta}_{3} \widehat{x}^{\prime 3}(T-\tau) \\
& +\left(\Omega^{2}-\varepsilon \sigma\right) \widehat{x}+\varepsilon \widehat{\beta} \widehat{x}^{3}=\varepsilon f \cos \Omega T .
\end{aligned}
$$

For simplicity, assuming a two-scale expansion of (9) as

$$
\begin{gathered}
\widehat{x}(\tau)=x_{0}\left(T_{0}, T_{1}\right)+\varepsilon x_{1}\left(T_{0}, T_{1}\right)+O\left(\varepsilon^{2}\right), \\
T_{n}=\varepsilon^{n} T, \quad n=0,1
\end{gathered}
$$


and using the following differential operators [22]

$$
\begin{gathered}
\frac{d}{d T}=\frac{\partial}{\partial T_{0}}+\varepsilon \frac{\partial}{\partial T_{1}}+O\left(\varepsilon^{2}\right)=D_{0}+\varepsilon D_{1}+O\left(\varepsilon^{2}\right) \\
\frac{d^{2}}{d T^{2}}=D_{0}^{2}+2 \varepsilon D_{0} D_{1}+O\left(\varepsilon^{2}\right) .
\end{gathered}
$$

Substituting (10) and (11) into (9) and equating the coefficients of the same power of $\varepsilon$, the following equations can be obtained:

$$
\begin{gathered}
D_{0}^{2} x_{0}+\Omega^{2} x_{0}=0 \\
D_{0}^{2} x_{1}+\Omega^{2} x_{1}=-2 D_{0} D_{1} x_{0}-2 \widehat{\xi}_{1} D_{0} x_{0} \\
-2 \widehat{\xi}_{3}\left[D_{0} x_{0}\left(T_{0}-\tau\right)\right]^{3}-\widehat{\beta} x_{0}^{3} \\
+\sigma x_{0}+f \cos \Omega T_{0} .
\end{gathered}
$$

The solution of (12a) can be written in the form

$$
\begin{gathered}
x_{0}\left(T_{0}, T_{1}\right)=A\left(T_{1}\right) e^{j \Omega T_{0}}+c c, \\
A\left(T_{1}\right)=\frac{1}{2} a\left(T_{1}\right) e^{j \Phi\left(T_{1}\right)},
\end{gathered}
$$

where $c c$ denotes the conjugate term and the amplitude $a$ and phase $\Phi$ are functions of the slow time scale $T_{1}$. Substituting (13a) into (12b) and removing secular terms from (12b) require

$$
\begin{aligned}
& -j 2 \Omega D_{1} A-j 2 \widehat{\xi}_{1} \Omega A-3 \widehat{\beta} A^{2} \bar{A} \\
& -j 6 \widehat{\zeta}_{3} \Omega^{3} A^{2} \bar{A} e^{-j \Omega \tau}+\sigma A+\frac{f}{2}=0 .
\end{aligned}
$$

Substituting (13b) into (14) and separating the real and imaginary terms, the following equations can be obtained:

$$
\begin{aligned}
a^{\prime} & =-\frac{1}{\Omega}\left(\Omega \widehat{\zeta}_{1} a+\frac{3}{4} \widehat{\zeta}_{3} \Omega^{3} a^{3} \cos \Omega \tau+\frac{f}{2} \sin \Phi\right), \\
a \Phi^{\prime} & =\frac{1}{\Omega}\left(-\frac{1}{2} \sigma a+\frac{3}{8} \widehat{\beta} a^{3}+\frac{3}{4} \widehat{\zeta}_{3} \Omega^{3} a^{3} \sin \Omega \tau-\frac{f}{2} \cos \Phi\right) .
\end{aligned}
$$

The steady state solutions of (9) for the primary resonance corresponding to the fixed points are given as

$$
\begin{array}{r}
\Omega \widehat{\zeta}_{1} a+\frac{3}{4} \widehat{\zeta}_{3} \Omega^{3} a^{3} \cos \Omega \tau=-\frac{f}{2} \sin \Phi \\
-\frac{1}{2} \sigma a+\frac{3}{8} \widehat{\beta} a^{3}+\frac{3}{4} \widehat{\zeta}_{3} \Omega^{3} a^{3} \sin \Omega \tau=\frac{f}{2} \cos \Phi .
\end{array}
$$

Then the amplitude-frequency and phase-frequency relationships using the original structural parameter can be given by

$$
\begin{aligned}
& \left(2 \Omega \zeta_{1} a+\frac{3}{2} \zeta_{3} \Omega^{3} a^{3} \cos \Omega \tau\right)^{2} \\
& \quad+\left(\left(\Omega^{2}-1\right) a-\frac{3}{4} \beta a^{3}-\frac{3}{2} \zeta_{3} \Omega^{3} a^{3} \sin \Omega \tau\right)^{2}=f_{1}^{2} \\
& \tan \Phi=\frac{\left(\Omega^{2}-1\right) a-(3 / 4) \beta a^{3}-(3 / 2) \zeta_{3} \Omega^{3} a^{3} \sin \Omega \tau}{2 \Omega \zeta_{1} a+(3 / 2) \zeta_{3} \Omega^{3} a^{3} \cos \Omega \tau}
\end{aligned}
$$

From (17a), it can be seen that the primary resonance of the controlled system is greatly influenced by the nondimensional feedback gain $\zeta_{3}$ and time delay $\tau$ when the other structural parameters and excitation force amplitude are chosen. The effects of these two parameters on the primary resonance can be seen by defining the equivalent damping ratio and equivalent resonance frequency as follows:

$$
\begin{gathered}
\zeta_{\mathrm{eq}}=\zeta_{1}+\frac{3}{4} \zeta_{3} \Omega^{2} a^{2} \cos \Omega \tau, \\
w_{\mathrm{eq}}^{2}=1+\frac{3}{4} \beta a^{2}+\frac{3}{2} \zeta_{3} \Omega^{3} a^{2} \sin \Omega \tau .
\end{gathered}
$$

Figure 3 shows the amplitude-frequency and phasefrequency relationships of the primary resonance for the controlled system under different feedback gain $\zeta_{3}$ without time delay. When the time delay $\tau=0$, the feedback gain $\zeta_{3}$ only appears in the equivalent damping ratio term and (18a) and (18b) simplify into

$$
\begin{gathered}
\zeta_{\mathrm{eq}}=\zeta_{1}+\frac{3}{4} \zeta_{3} \Omega^{2} a^{2}, \\
w_{\mathrm{eq}}^{2}=1+\frac{3}{4} \beta a^{2} .
\end{gathered}
$$

The effect of the feedback gain $\zeta_{3}$ on the primary resonance can be clearly seen in Figure 3 . When the linear damping ratio $\zeta_{1}$ is small and the system is uncontrolled, the peak amplitude of the primary resonance takes a larger value and the jump phenomenon occurs. When the feedback gain $\zeta_{3}$ increases, the equivalent damping ratio becomes larger, the peak amplitude of the primary resonance decreases, the equivalent resonance frequency decreases, and the jump phenomenon can be avoided.

When the time delay $\tau$ exists in the controlled system, the following inequalities of the equivalent damping ratio and equivalent resonance frequency can be obtained:

$$
\zeta_{1}-\frac{3}{4} \zeta_{3} \Omega^{2} a^{2} \leq \zeta_{\mathrm{eq}}=\zeta_{1}+\frac{3}{4} \zeta_{3} \Omega^{2} a^{2} \cos \Omega \tau \leq \zeta_{1}+\frac{3}{4} \zeta_{3} \Omega^{2} a^{2},
$$



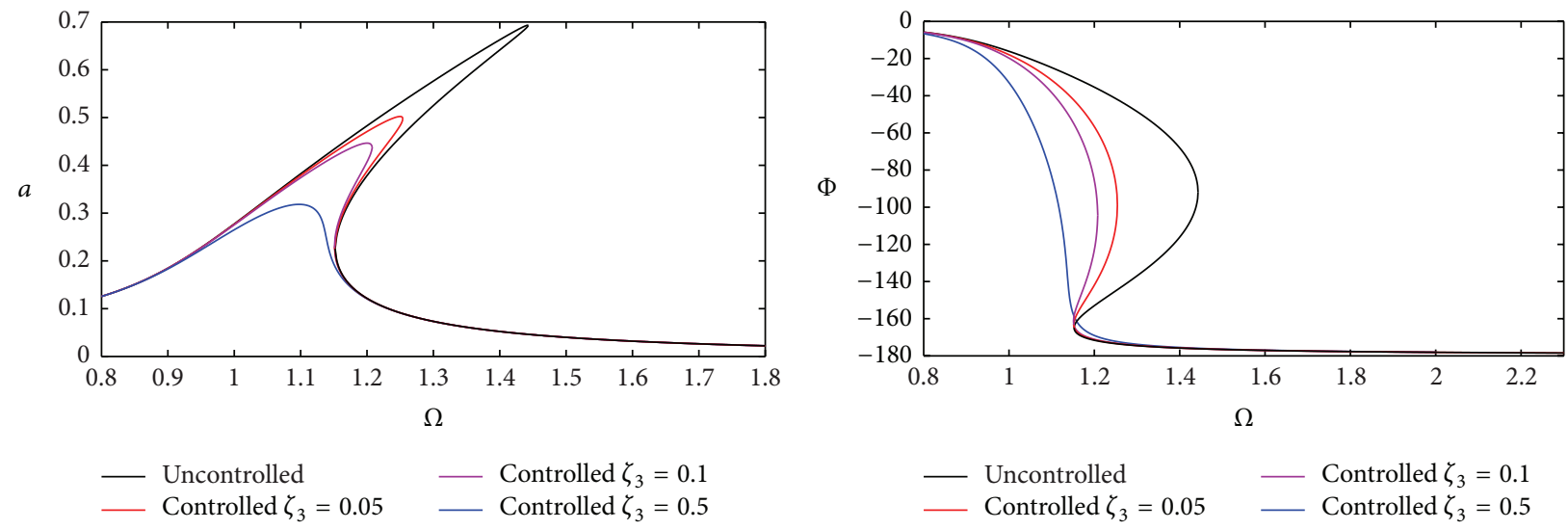

FIGURE 3: Amplitude-frequency and phase-frequency relationships of the primary resonance for the controlled system under different feedback gain $\zeta_{3}$ without time delay $\left(\zeta_{1}=0.025, f_{1}=0.05, k=1\right.$, and $\left.\hat{\lambda}=0.4\right)$.

$$
\begin{aligned}
1+\frac{3}{4} \beta a^{2}-\frac{3}{2} \zeta_{3} \Omega^{3} a^{2} & \leq w_{\text {eq }}^{2} \\
& =1+\frac{3}{4} \beta a^{2}+\frac{3}{2} \zeta_{3} \Omega^{3} a^{2} \sin \Omega \tau \\
& \leq 1+\frac{3}{4} \beta a^{2}+\frac{3}{2} \zeta_{3} \Omega^{3} a^{2}
\end{aligned}
$$

Obviously, compared to the controlled system without time delay, when time delay $\tau$ exists, it can decrease the equivalent damping ratio; then the peak amplitude increases; the equivalent resonance frequency can also increase which leads to an undesirable isolation performance. In the primary resonance region, assuming that $\Omega \approx 1$, the equivalent damping ratio and equivalent resonance frequency can be approximated as

$$
\begin{gathered}
\zeta_{\text {eq }}=\zeta_{1}+\frac{3}{4} \zeta_{3} a^{2} \cos \tau, \\
w_{\text {eq }}^{2}=1+\frac{3}{4} \beta a^{2}+\frac{3}{2} \zeta_{3} a^{2} \sin \tau .
\end{gathered}
$$

Then the equivalent damping ratio and equivalent resonance frequency can be approximately considered as the periodic functions in $2 \pi$ periods. Based on this assumption, the effect of the time delay $\tau$ on the primary resonance can be clearly seen in Figure 4 . When the time delay $\tau$ lies in the ranges of $[0, \pi / 2)$ and $[3 \pi / 2,2 \pi)$, the equivalent damping ratio is larger than the linear damping ratio; then the peak amplitude and resonance frequency are smaller than the uncontrolled system which can achieve a better isolation performance in the primary resonance region; the equivalent damping ratio takes a major effect on the peak amplitude and resonance frequency in these two ranges. When the time delay $\tau$ lies in the range of $[\pi / 2, \pi]$, the equivalent damping ratio is smaller than the linear damping ratio and the equivalent resonance frequency is larger resulting in a poorer isolation performance in the primary resonance region than the uncontrolled system. When the time delay $\tau$ lies in the range of $(\pi, 3 \pi / 2)$, although the equivalent damping ratio is smaller than the linear damping ratio, the equivalent resonance frequency is smaller and takes a major effect on the peak amplitude and resonance frequency; the peak amplitude and resonance frequency are smaller which can achieve a better isolation performance in the primary resonance region compared to the uncontrolled system.

Figure 4 also shows the numerical results obtained by solving (7) using the fourth order Runge-Kutta method; the analytic method gives very well results compared with the numerical results. But when the time delay $\tau$ takes some special values, a closed-loop response curve [23] emerges above the primary resonance because multiple solutions exist in these frequency bands; some closed-loop response curves can be estimated by numerical results and some cannot be which are due to their stability. In Figures 4(c), 4(d), 4(e), and 4(i), some parts of the upper branch of the primary resonance cannot also be estimated by numerical results which are also due to their stability. Stability analysis of the controlled system will be investigated in the stability analysis part.

As can be clearly seen in Figures 3 and 4, the feedback gain $\zeta_{3}$ mostly influences the vibration amplitude of the controlled system around the resonance frequency band. When the feedback gain $\zeta_{3}$ changes, the vibration amplitudes of the controlled system in the low and high frequency bands are almost the same as the uncontrolled system. Figure 5 shows the effect of the time delay $\tau$ on the primary resonance for the controlled system with different feedback gain $\zeta_{3}$ around the resonance frequency band. In this case only one single vibration amplitude is considered, the frequency $\Omega$ is chosen as 1 and 1.1, respectively, and the stability analysis of the vibration amplitude is also investigated in the stability analysis part.

The vibration amplitude is a periodic function of time delay $\tau$ which can be seen in (17a). In the first half period, the vibration amplitude decreases with the increase of feedback gain $\zeta_{3}$, while in the latter half period the vibration amplitude first increases and then decreases with the increase of feedback gain $\zeta_{3}$. Figure 6 shows the corresponding amplitude-frequency relationships of the primary resonance for the controlled system with different feedback gain $\zeta_{3}$. When $\tau=\pi / 4$ lies in the range of the first half period, with the increase of feedback gain $\zeta_{3}$, the vibration amplitude around 


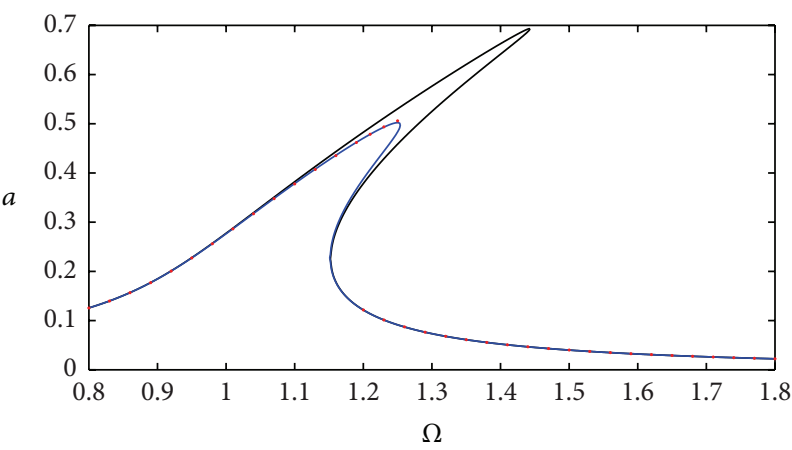

(a) $\tau=0$

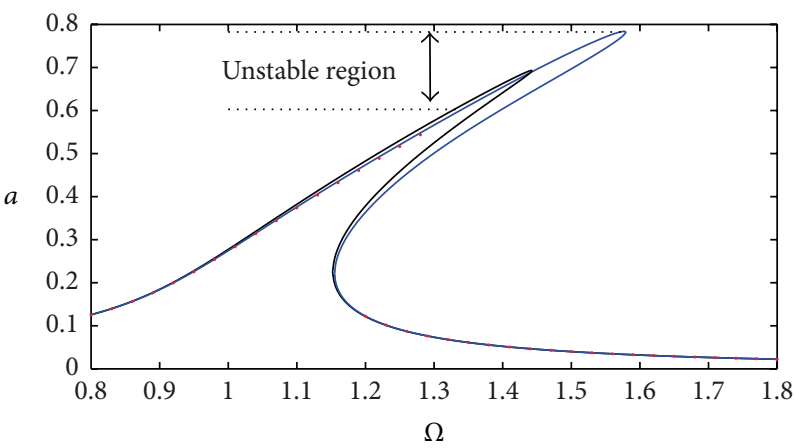

(c) $\tau=\pi / 2$

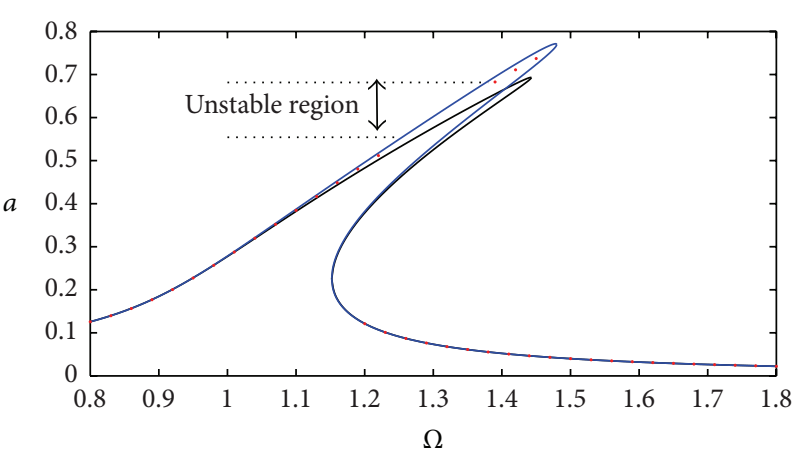

(e) $\tau=\pi$

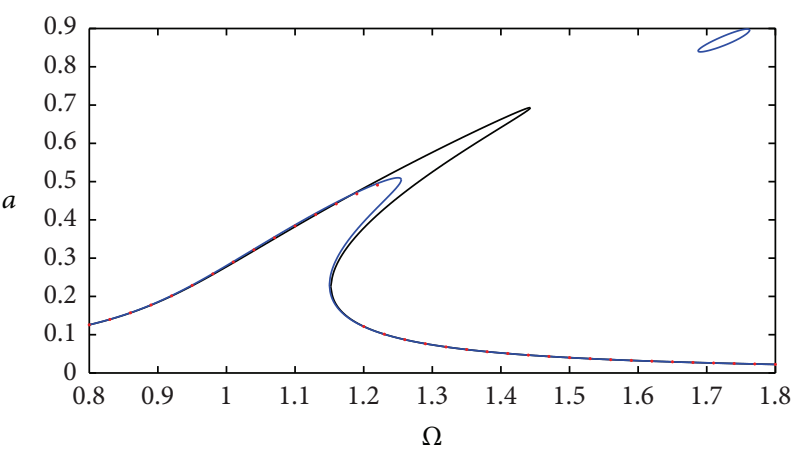

Uncontrolled
Controlled

- Numerical

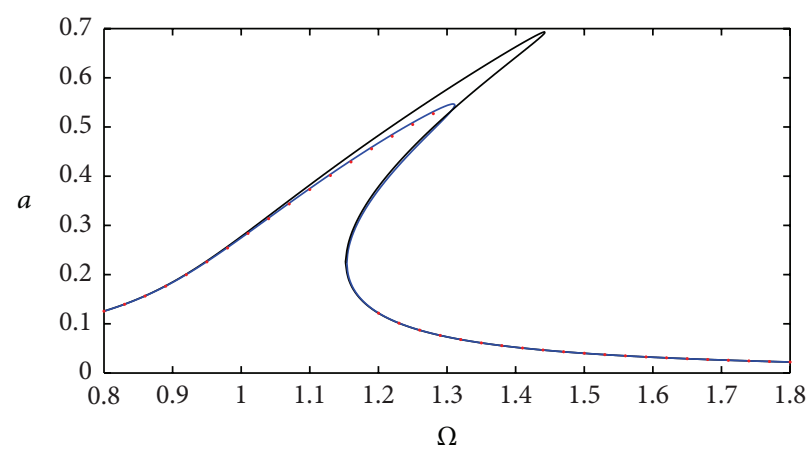

(b) $\tau=\pi / 4$

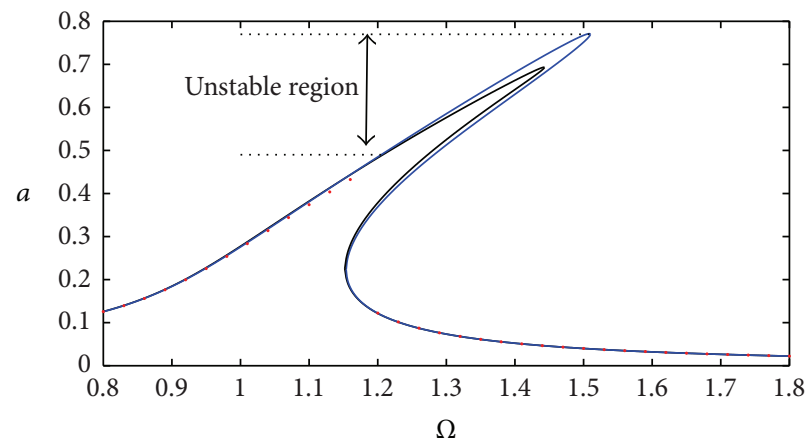

(d) $\tau=3 \pi / 4$

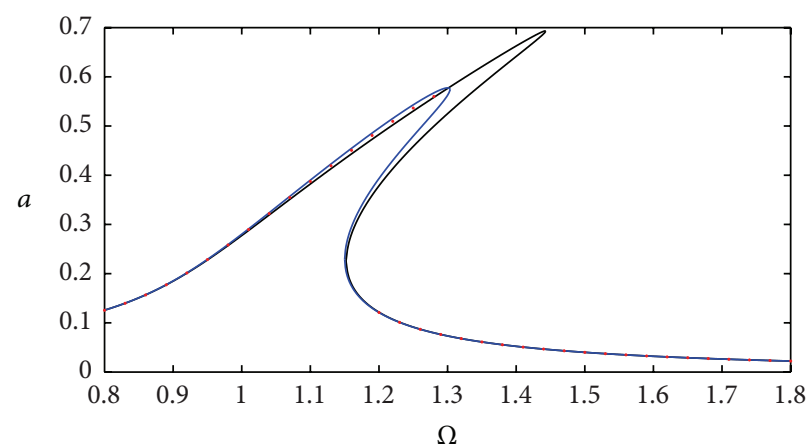

(f) $\tau=5 \pi / 4$

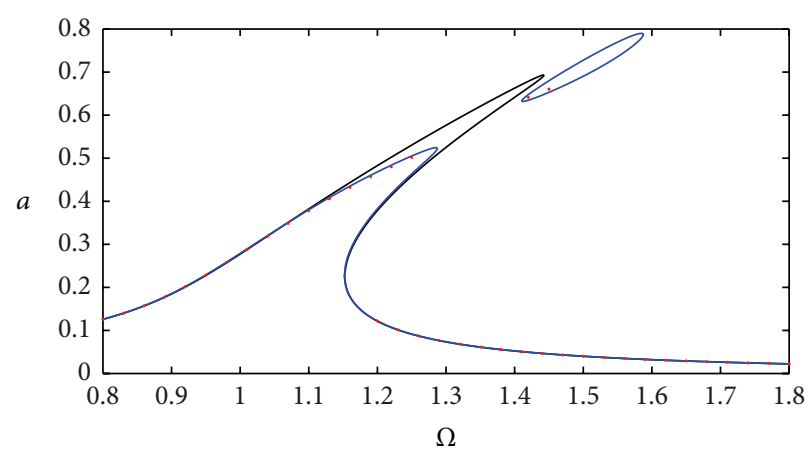

$$
\begin{aligned}
& \text { Uncontrolled } \\
& \text { Controlled } \\
& \text { - Numerical }
\end{aligned}
$$

(h) $\tau=7 \pi / 4$

(g) $\tau=3 \pi / 2$

Figure 4: Continued. 

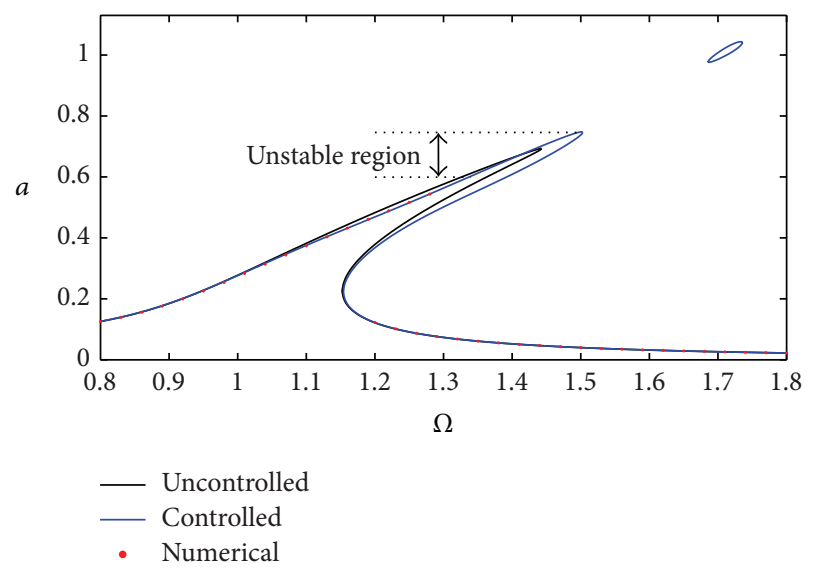

(i) $\tau=2 \pi$

FIGURE 4: Amplitude-frequency relationships of the primary resonance for the controlled system with different time delay $\tau\left(\zeta_{1}=0.025\right.$, $\zeta_{3}=0.05, f_{1}=0.05, k=1$, and $\left.\hat{\lambda}=0.4\right)$.

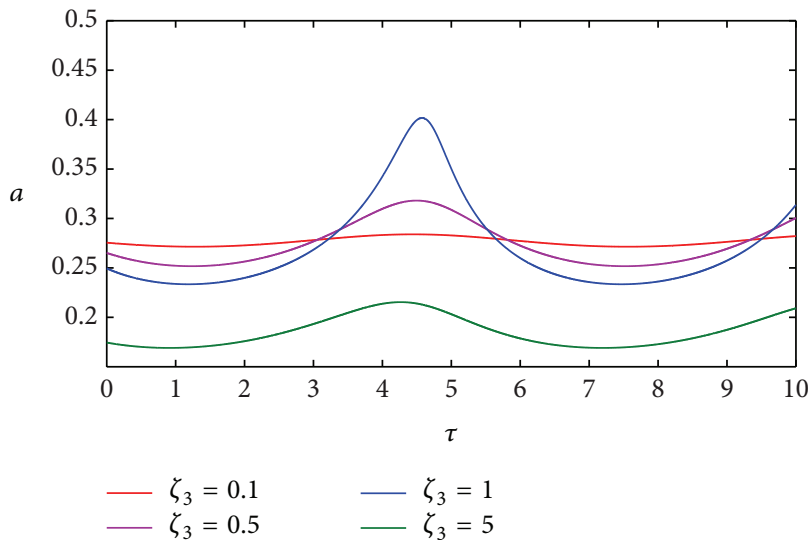

(a) $\Omega=1$
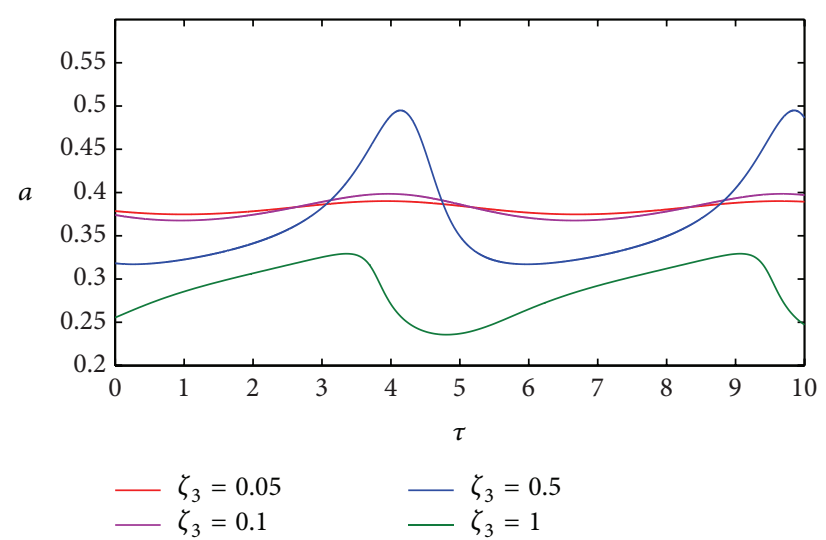

(b) $\Omega=1.1$

FigURE 5: Effect of the time delay $\tau$ on the primary resonance for the controlled system with different feedback gain $\zeta_{3}\left(\zeta_{1}=0.025, f_{1}=0.05\right.$, $k=1$, and $\hat{\lambda}=0.4)$.

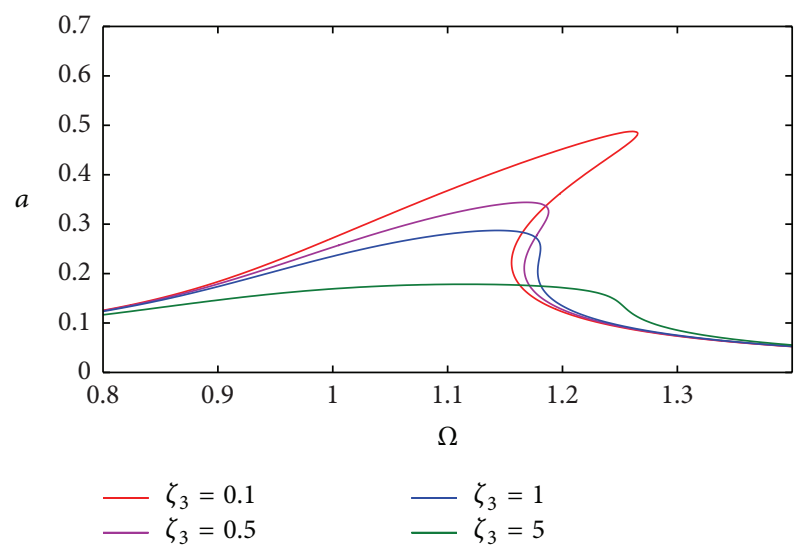

(a) $\tau=\pi / 4$

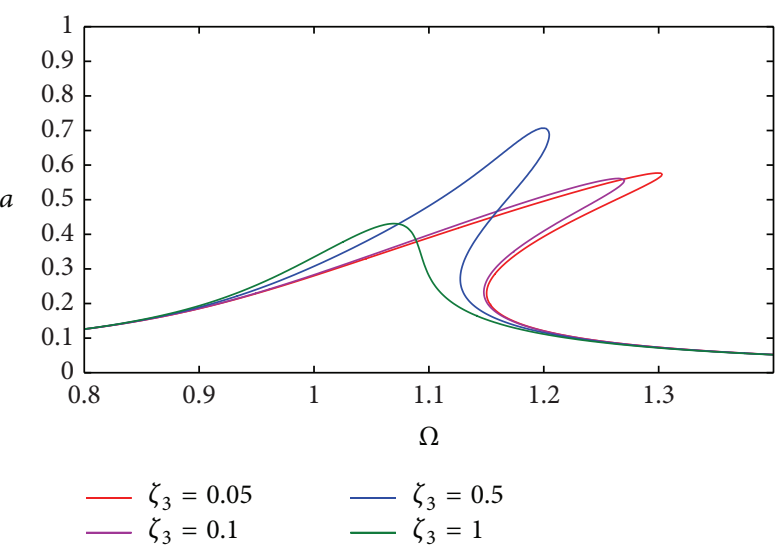

(b) $\tau=5 \pi / 4$

FIGURE 6: Amplitude-frequency relationships of the primary resonance for the controlled system with different feedback gain $\zeta_{3}\left(\zeta_{1}=0.025\right.$, $f_{1}=0.05, k=1$, and $\left.\widehat{\lambda}=0.4\right)$. 
the resonance frequency band decreases and jump phenomenon can be avoided. When $\tau=5 \pi / 4$ lies in the range of the latter half period, with the increase of feedback gain $\zeta_{3}$, the vibration amplitude around the resonance frequency band first increases and then decreases, and jump phenomenon can also be avoided when feedback gain $\zeta_{3}$ increases to a larger value.

3.2. Jump Phenomenon Analysis. From Figures 3 and 4, it can be seen that jump phenomenon can occur in the primary resonance of the controlled system when the structural parameters and excitation force amplitude are chosen as some specific values. The primary response amplitude decreases at jump-down frequency and increases at jump-up frequency. Jump phenomenon can be detrimental and can probably cause extensive physical damage to the controlled system due to the large amplitude changes occurring at jump-down or jump-up frequency. So it is beneficial to determine the critical surface of the structural parameters which lead to no jump phenomenon, and the jump frequencies should be determined when jump phenomenon occurs. The jump phenomenon analysis of the controlled system without and with time delay is investigated, respectively, in this part.

The amplitude-frequency function of the controlled system without time delay can be simplified into

$$
\left(2 \Omega \zeta_{1} a+\frac{3}{2} \zeta_{3} \Omega^{3} a^{3}\right)^{2}+\left(\left(\Omega^{2}-1\right) a-\frac{3}{4} \beta a^{3}\right)^{2}=f_{1}^{2} .
$$

Equation (22) can be written as a polynomial function in amplitude $a$ or frequency $\Omega$ as follows:

$$
\begin{gathered}
H(\Omega, a)=p_{1} a^{6}+q_{1} a^{4}+r_{1} a^{2}+s_{1}, \\
p_{1}=\frac{9}{16} \beta^{2}+\frac{9}{4} \zeta_{3}^{2} \Omega^{6}, \\
q_{1}=6 \zeta_{1} \zeta_{3} \Omega^{4}-\frac{3}{2} \beta\left(\Omega^{2}-1\right), \\
r_{1}=4 \zeta_{1}^{2} \Omega^{2}+\left(\Omega^{2}-1\right)^{2}, \\
s_{1}=-f_{1}^{2}, \\
\Omega, a)=p_{2} \Omega^{6}+q_{2} \Omega^{4}+r_{2} \Omega^{2}+s_{2}, \\
p_{2}=\frac{9}{4} \zeta_{3}^{2} a^{6}, \\
q_{2}=6 \zeta_{1} \zeta_{3} a^{4}+a^{2}, \\
r_{2}=-\frac{3}{2} \beta a^{4}+\left(4 \zeta_{1}^{2}-2\right) a^{2}, \\
s_{2}=\frac{9}{16} \beta^{2} a^{6}+\frac{3}{2} \beta a^{4}+a^{2}-f_{1}^{2} .
\end{gathered}
$$

It is inconvenient to solve the amplitude $a$ or frequency $\Omega$ in an analytic expression since $(23 \mathrm{a})-(23 \mathrm{j})$ can be treated as a cubic function of amplitude $a^{2}$ or frequency $\Omega^{2}$; it is difficult to determine the critical surface of the structural parameters and jump frequencies based on these analytic expressions. So the method of Sylvester resultant [24] is used in this paper. Assuming two polynomials $f(x)$ and $g(x)$ as

$$
\begin{aligned}
& f(x)=\sum_{i=0}^{n} a_{i} x^{i}, \quad a_{n} \neq 0, \\
& g(x)=\sum_{i=0}^{m} b_{i} x^{i}, \quad b_{m} \neq 0 .
\end{aligned}
$$
[25]

The Sylvester resultant of $f(x)$ and $g(x)$ can be obtained

$$
\Re(f, g)=\left|\begin{array}{ccccccccc}
a_{n} & a_{n-1} & \cdots & \cdots & a_{1} & a_{0} & 0 & \cdots & 0 \\
0 & a_{n} & a_{n-1} & \cdots & \cdots & a_{1} & a_{0} & \cdots & 0 \\
\cdots & \cdots & \cdots & & & & \cdots & \cdots & \cdots \\
0 & \cdots & 0 & a_{n} & a_{n-1} & \cdots & \cdots & \cdots & a_{0} \\
b_{m} & b_{m-1} & \cdots & b_{1} & b_{0} & 0 & \cdots & \cdots & 0 \\
0 & b_{m} & b_{m-1} & \cdots & b_{1} & b_{0} & 0 & \cdots & 0 \\
\cdots & \cdots & \cdots & & & & \cdots & \cdots & \cdots \\
0 & \cdots & \cdots & 0 & b_{m} & b_{m-1} & \cdots & \cdots & b_{0}
\end{array}\right| .
$$

A necessary and sufficient condition for two polynomials $f(x)$ and $g(x)$ to have a common root is that the Sylvester resultant $\mathfrak{R}(f, g)=0$ [26].

When the excitation force amplitude takes a smaller value for chosen structural parameter, jump phenomenon does not occur and the amplitude-frequency curve is single-valued which is similar to the linear harmonic excited system; that is, there exists a unique value of amplitude $a$ for each value of frequency $\Omega$. When the excitation force amplitude increases to a larger value, jump phenomenon can occur due to the existence of cubic nonlinear terms; there exist multiple values of amplitude $a$ for each value of frequency $\Omega$ in a specific frequency band. Denote $f_{1 \mathrm{cr}}$ as the critical value of the excitation force amplitude, that is the maximum excitation force amplitude which leads to no jump phenomenon. Denoting $\left(\Omega_{\mathrm{cr}}, a_{\mathrm{cr}}\right)$ as the infection point in the amplitude-frequency curve when $f_{1}=f_{1 \mathrm{cr}}$, then the function $H\left(\Omega_{\mathrm{cr}}, a\right)$ has three positive real roots equal to $a_{\mathrm{cr}}$. Assuming the functions $H^{\prime}(\Omega, a)$ and $H^{\prime \prime}(\Omega, a)$ as the derivative and second derivative of the function $H(\Omega, a)$ with respect to the amplitude $a$, respectively, then the function $H^{\prime}\left(\Omega_{c r}, a\right)$ has two positive real roots equal to $a_{\mathrm{cr}}$ and the function $H^{\prime \prime}\left(\Omega_{\mathrm{cr}}, a\right)$ has one positive real root equal to $a_{\mathrm{cr}}$. According to the definition of Sylvester resultant, the resultant $\mathfrak{R}\left(H^{\prime}, H^{\prime \prime}\right)$ should be equal to zero at the infection point $\left(\Omega_{\mathrm{cr}}, a_{\mathrm{cr}}\right)$.

Combining (23a)-(23j) and (25), the following equation can be obtained:

$$
G\left(a_{\mathrm{cr}}\right)=\left.\Re\left(H^{\prime}, H^{\prime \prime}\right)\right|_{a=a_{\mathrm{cr}}}=\sum_{i=0}^{10} b_{i} a_{\mathrm{cr}}^{i}=0,
$$

where the definitions of the coefficients $b_{i}$ are given in Appendix. Using (26), $\Omega_{\text {cr }}$ can be eliminated and can obtain a polynomial equation in $a_{\mathrm{cr}}$. Then the value of $a_{\mathrm{cr}}$ can be obtained 
numerically. Of the ten roots of $G\left(\Omega_{\mathrm{cr}}\right)=0$, there are two real and positive roots and the value of $a_{\mathrm{cr}}$ should be taking the smaller value. The critical excitation frequency $\Omega_{\mathrm{cr}}$ can be given by substituting $a_{\mathrm{cr}}$ into the function $H^{\prime \prime}\left(\Omega_{\mathrm{cr}}, a\right)$. Once the critical excitation frequency $\Omega_{\mathrm{cr}}$ and critical amplitude $a_{\mathrm{cr}}$ are known, the critical excitation force amplitude $f_{1 \mathrm{cr}}$ can be obtained by using (23a) or (23f).

When the excitation force amplitude $f_{1}>f_{1 \mathrm{cr}}$, jump phenomenon occurs; it is necessary to determine the jumpup and jump-down frequencies. Denoting $\left(\Omega_{*}, a_{*}\right)$ as the jump points in the amplitude-frequency curve, then the function $H\left(\Omega_{*}, a\right)$ has two positive real roots equal to $a_{*}$, which requires that the resultant $\mathfrak{R}\left(H, H^{\prime}\right)$ should be equal to zero at the jump points $\left(\Omega_{*}, a_{*}\right)$.

Combining (23a)-(23j) and (25), the following equation can be obtained:

$$
G\left(a_{*}\right)=\left.\Re\left(H, H^{\prime}\right)\right|_{a=a_{*}}=\sum_{i=0}^{18} c_{i} a_{*}^{i}=0,
$$

where the definitions of the coefficients $c_{i}$ are given in Appendix. Using (27), $\Omega_{*}$ can be eliminated and can obtain a polynomial equation in $a_{*}$. Then the value of $a_{*}$ can be obtained numerically. Of the eighteen roots of $G\left(\Omega_{\mathrm{cr}}\right)=0$, there are two real and positive roots; the smaller value corresponds to the amplitude of jump-up frequency and the larger value corresponds to the amplitude of jump-down frequency. The jump frequencies can be obtained by substituting $a_{*}$ into the function $H^{\prime}\left(\Omega_{\mathrm{cr}}, a\right)$. But for each value of $a_{*}$, there are two real and positive values of $\Omega_{*}$, one of which is spurious. To investigate which one is spurious, substitute $\Omega_{*}$ into the function $H\left(\Omega_{*}, a\right)$ and check whether there are two real and positive values equal to $a_{*}$ or not. If there are none, then this particular value of $\Omega_{*}$ is spurious and should be discarded.

Amplitude-frequency relationship of the primary resonance for the controlled system under different excitation force amplitude without time delay is shown in Figure 7. Using the method of Sylvester resultant, the critical excitation force amplitude $f_{1 \mathrm{cr}}=0.01102$. The infection point $\left(\Omega_{\mathrm{cr}}, a_{\mathrm{cr}}\right)$ can be obtained as $(1.0485,0.1698)$ which can be seen in Figure 7 denoted by black asterisk point. As discussed previously, when the excitation force amplitude $f_{1}=0.03>$ $f_{1 \mathrm{cr}}$, jump phenomenon occurs; the jump points $\left(\Omega_{*}, a_{*}\right)$ can also be determined by the method of Sylvester resultant. The jump-up points and jump-down points can be obtained as $(1.108,0.1945)$ and $(1.163,0.3891)$, respectively, which can be seen in Figure 7 denoted by black dot points. Figure 8 shows the critical surface of the structural parameters which lead to no jump phenomenon. It is apparent that the reasonable structural parameter combinations should lie under the critical surface. Figure 8 also shows the partial contours of the critical surface; the direction of the arrow indicates the reasonable structural parameter combinations which lead to no jump phenomenon.

The jump phenomenon analysis of the controlled system with time delay is then considered. Because the trigonometric functions exist in the amplitude-frequency function of the primary resonance, the method of Sylvester resultant cannot be used in this case. The critical boundary which leads to no

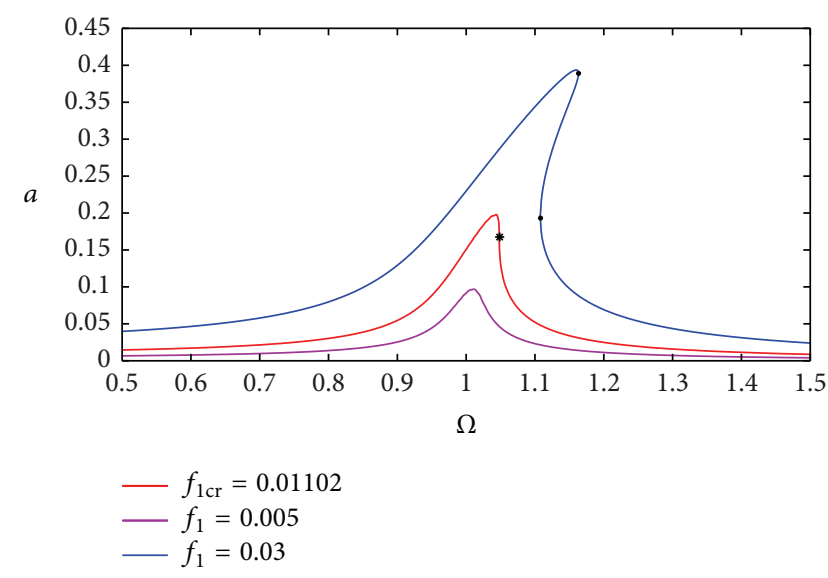

FIGURE 7: Amplitude-frequency relationship of the primary resonance for the controlled system under different excitation force amplitude without time delay $\left(\zeta_{1}=0.025, \zeta_{3}=0.05, k=1\right.$, and $\widehat{\lambda}=0.4)$.

jump phenomenon and jump frequencies will be investigated by using the optimization method.

For the controlled system with time delay, when there are no points in the amplitude-frequency curves satisfying the condition $d \Omega / d a=0$, jump phenomenon does not occur [27]. But when there are at least two points in the amplitudefrequency curves satisfying the condition $d \Omega / d a=0$, the jump phenomenon can occur. So the critical condition is that there is only one point in the amplitude-frequency curves satisfying the condition $d \Omega / d a=0$. Differentiating both sides of (17a) with respect to $a$ and substituting the condition $d \Omega / d a=0$ in it, the critical function can be given as

$$
\begin{gathered}
\left(2 \Omega \zeta_{1}+\frac{3}{2} \zeta_{3} \Omega^{3} a^{2} \cos \Omega \tau\right)\left(2 \Omega \zeta_{1}+\frac{9}{2} \zeta_{3} \Omega^{3} a^{2} \cos \Omega \tau\right) \\
+\left(\left(\Omega^{2}-1\right)-\frac{3}{4} \beta a^{2}-\frac{3}{2} \zeta_{3} \Omega^{3} a^{2} \sin \Omega \tau\right) \\
\cdot\left(\left(\Omega^{2}-1\right)-\frac{9}{4} \beta a^{2}-\frac{9}{2} \zeta_{3} \Omega^{3} a^{2} \sin \Omega \tau\right)=0
\end{gathered}
$$

Combining the critical function equation (28) and the amplitude-frequency function equation (17a), the critical boundary and jump frequencies can be achieved.

An example of how to determine the critical excitation force amplitude $f_{1 \mathrm{cr}}$ by using the optimization method is presented when the other structural parameters are chosen. The Levenberg-Marquardt (LM) optimization method is considered in this paper. Denoting the critical function equation (28) and the amplitude-frequency function equation (17a) as the objective functions and defining frequency $\Omega$ and amplitude $a$ as the independent variable. Set up the convergence index $\eta$ as $1 e-10$ and the maximum number of iterations $N$ as 2000 . For a given critical excitation force amplitude $f_{1 \mathrm{cr}}$, the frequency $\Omega$ and amplitude $a$ can be determined by using the LM optimization method to minimize the objective functions; then the value of objective functions can be obtained. 

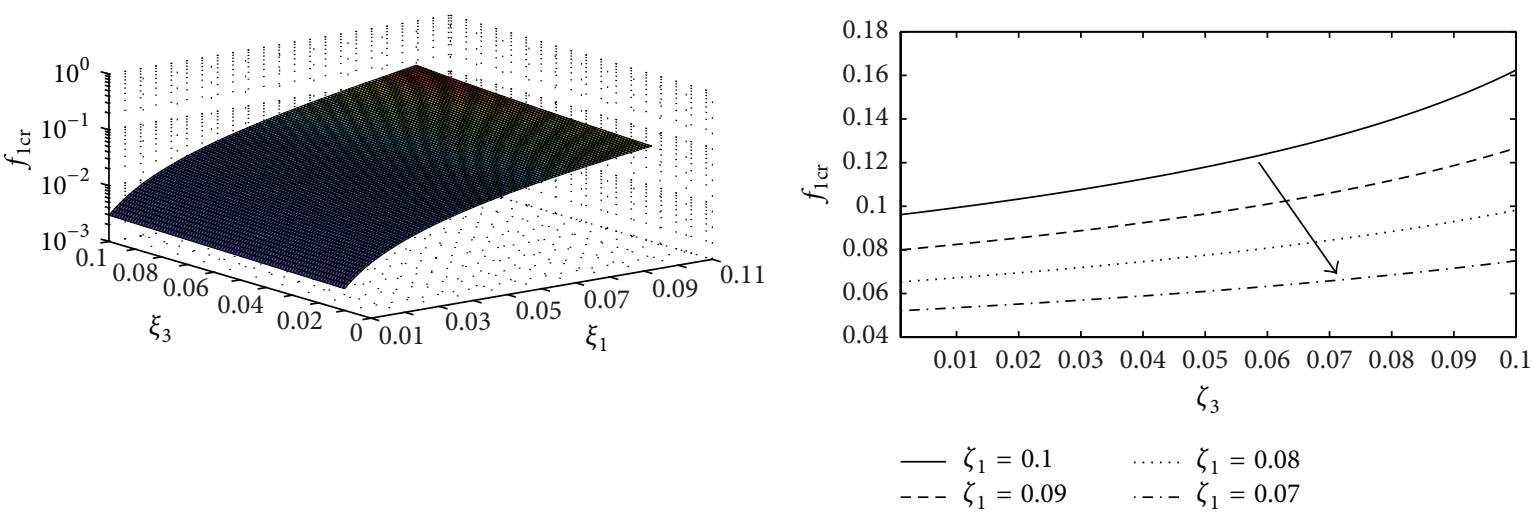

FIGURE 8: Critical surface and contours of the critical surface of the controlled system without time delay $(k=1$ and $\hat{\lambda}=0.4)$.

Substituting the initial guess value of critical excitation force amplitude $f_{1 \text { icr }}$ into the objective functions and using the LM optimization method, when the initial value of $f_{\text {licr }}$ is chosen as a larger one, the value of the objective functions is smaller than the convergence index $\eta$ which indicates the value of $f_{\text {licr }}$ is larger and jump phenomenon occurs; the frequency $\Omega$ and amplitude $a$ can be obtained as the jump frequency and the amplitude of jump frequency, respectively, decreasing the value of $f_{\text {licr }}$ slowly until the value of the objective functions is larger than the convergence index $\eta$ for the first time; then the critical excitation force amplitude $f_{1 \mathrm{cr}}$ can be obtained; when the initial value of $f_{1 \text { icr }}$ is chosen as a smaller one, the value of the objective functions is larger than the convergence index $\eta$ which indicates the value of $f_{1 \text { icr }}$ is smaller and jump phenomenon does not occur; the obtained frequency $\Omega$ and amplitude $a$ are spurious and should be discarded, increasing the value of $f_{\text {licr }}$ slowly until the value of the objective functions is smaller than the convergence index $\eta$ for the first time; then the critical excitation force amplitude $f_{\text {lcr }}$ can be obtained. The solution process for determining the critical excitation force amplitude $f_{\text {licr }}$ is an iterative process.

The critical border of the controlled system with time delay $\tau=\pi / 4$ is shown in Figure 9. Using the LM optimization method, the critical excitation force amplitude $f_{1 \mathrm{cr}}=$ 0.01046 . When the excitation force amplitude $f_{1}=f_{1 \mathrm{cr}}$, there is only one point in the amplitude-frequency curves satisfying the condition $d \Omega / d a=0$ which corresponds to the boundary. When the excitation force amplitude $f_{1}>f_{1 \mathrm{cr}}$, there are two points in the amplitude-frequency curves satisfying the condition $d \Omega / d a=0$ which implies the jump phenomenon. When the jump phenomenon occurs, the jump points can be determined conveniently by using the LM optimization method. Figure 10 shows the amplitude-frequency relationship of the primary resonance for the controlled system under different excitation force amplitude with time delay $\tau=\pi / 4$. When the excitation force amplitude $f_{1}=0.03>f_{1 \mathrm{cr}}$, the jump-up points and jump-down points can be obtained as $(1.1095,0.1914)$ and $(1.1973,0.4129)$, respectively, which can be seen in Figure 10 denoted by black dot points.

The other critical values of structural parameters, for example, damping ratio $\zeta_{1}$ and feedback gain $\zeta_{3}$, can be determined just as the critical excitation force amplitude case, but the solution process is complicated which can be seen in the above part, so it is no longer discussed here.

3.3. Stability Analysis. To analyze the stability of the primary response of the controlled system, linearizing (15a) and (15b) with respect to $a$ and $\Phi$ gives

$$
\begin{aligned}
D_{1} \Delta a= & -\left(\widehat{\zeta}_{1}+\frac{9}{4} \widehat{\zeta}_{3} \Omega^{2} a^{2} \cos \Omega \tau\right) \Delta a-\frac{f}{2} \cos \Phi \Delta \Phi \\
D_{1} \Delta \Phi= & \left(\frac{3}{4 \Omega} \widehat{\beta} a+\frac{3}{2} \widehat{\zeta}_{3} \Omega^{2} a \sin \Omega \tau+\frac{f}{2 \Omega a^{2}} \cos \Phi\right) \Delta a \\
& +\frac{f}{2 \Omega a} \sin \Phi \Delta \Phi .
\end{aligned}
$$

The characteristic equation of (29a) and (29b) is given as

$\operatorname{det}\left|\begin{array}{cc}s+\widehat{\zeta}_{1}+\frac{9}{4} \widehat{\zeta}_{3} \Omega^{2} a^{2} \cos \Omega \tau & \frac{f}{2} \cos \Phi \\ -\left(\frac{3}{4 \Omega} \widehat{\beta} a+\frac{3}{2} \widehat{\zeta}_{3} \Omega^{2} a \sin \Omega \tau+\frac{f}{2 \Omega a^{2}} \cos \Phi\right) & s-\frac{f}{2 \Omega a} \sin \Phi\end{array}\right|=0$.

Equation (30) can be simplified using (16a) and (16b):

$$
\begin{gathered}
s^{2}-G_{1} s+G_{2}=0 \\
G_{1}=-2 \widehat{\zeta}_{1}-3 \widehat{\zeta}_{3} \Omega^{2} a^{2} \cos \Omega \tau \\
G_{2}=\left(\widehat{\zeta}_{1}+\frac{3}{4} \widehat{\zeta}_{3} \Omega^{2} a^{2} \cos \Omega \tau\right)\left(\widehat{\zeta}_{1}+\frac{9}{4} \widehat{\zeta}_{3} \Omega^{2} a^{2} \cos \Omega \tau\right) \\
+\frac{1}{\Omega^{2}}\left(\frac{\sigma}{2}-\left(\frac{3}{8} \widehat{\beta} a^{2}+\frac{3}{4} \widehat{\zeta}_{3} \Omega^{3} a^{2} \sin \Omega \tau\right)\right) \\
\cdot\left(\frac{\sigma}{2}-\left(\frac{9}{8} \widehat{\beta} a^{2}+\frac{9}{4} \widehat{\zeta}_{3} \Omega^{3} a^{2} \sin \Omega \tau\right)\right)
\end{gathered}
$$



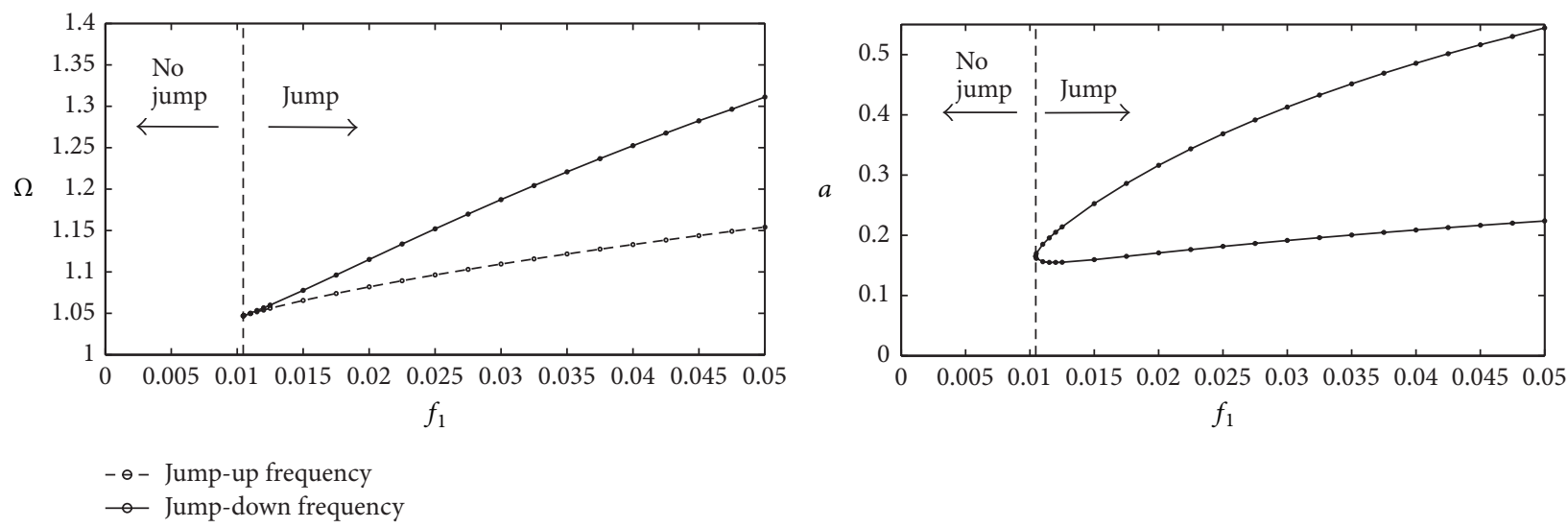

FIGURE 9: Critical border of the controlled system with time delay $\tau=\pi / 4\left(\zeta_{1}=0.025, \zeta_{3}=0.05, k=1\right.$, and $\left.\hat{\lambda}=0.4\right)$.

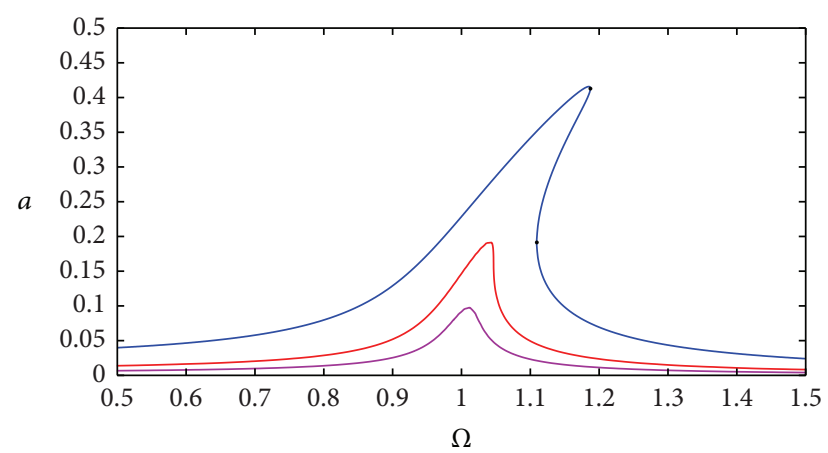

$$
\begin{aligned}
& -f_{\text {1cr }}=0.01046 \\
& -f_{1}=0.005 \\
& -f_{1}=0.03
\end{aligned}
$$

FIGURE 10: Amplitude-frequency relationship of the primary resonance for the controlled system under different excitation force amplitude with time delay $\tau=\pi / 4\left(\zeta_{1}=0.025, \zeta_{3}=0.05, k=1\right.$, and $\hat{\lambda}=0.4)$.

According to the Routh-Hurwitz criterion, the controlled system has stable primary response corresponding to stable node when $G_{1}<0$ and $G_{2}>0$, has unstable primary response corresponding to unstable node when $G_{1}>0$ and $G_{2}>0$, and has unstable primary response corresponding to saddle point when $G_{2}<0$. Actually, the critical condition $G_{1}=0$ indicates a pair of purely imaginary eigenvalues exist and hence a Hopf bifurcation occurs [16]. For the uncontrolled system $G_{1}=$ $-2 \widehat{\zeta}_{1}<0$ and controlled system without time delay $G_{1}=$ $-2 \widehat{\zeta}_{1}-3 \widehat{\zeta}_{3} \Omega^{2} a^{2}<0$, the Hopf bifurcation can be avoided.

The stability boundary of the primary resonance for the controlled system with different time delay is shown in Figure 11. The gray shaded unstable regions denoted by USR1 are determined by $G_{1}$ and the green shaded unstable regions denoted by USR 2 are determined by $G_{2}$. When time delay $\tau=\pi / 4, G_{1}>0$ in the given frequency band, and the unstable regions are determined by $G_{2}$. When time delay $\tau=\pi / 2$, the unstable regions are determined by both $G_{1}$ and $G_{2}$. The upper branch of the primary resonance is cut off by the boundary of USR1 determined by $G_{1}$; then these unstable responses cannot be obtained by numerical method which can be clearly seen in Figure 4(c). When time delay $\tau=7 \pi / 4$, a closed-loop response curve emerges above the primary resonance. It is observed that the lower branch of the closed-loop response curve is all covered by USR1 and USR2 and the upper branch of the closed-loop response curve is partially covered by USR 1 which can also be clearly seen in Figure 4(h) with using numerical method checked.

From (31a)-(31c), it can be seen that the stability boundary of the primary resonance for the controlled system is greatly influenced by the nondimensional feedback gain $\zeta_{3}$ and time delay $\tau$ when the other structural parameters and excitation force amplitude are chosen. Two cases are considered when investigating the effects of these two parameters on the stability of vibration amplitude for the controlled system. The first case is when only one single vibration amplitude exists and the second case is when three vibration amplitudes may exist in the controlled system.

Figure 12 shows the effect of the time delay $\tau$ on the stability of vibration amplitude for the controlled system with different feedback gain $\zeta_{3}$. The dashed line indicates the unstable vibration amplitude. When $\Omega=1.1$, only one single vibration amplitude exists in the controlled system. When feedback gain $\zeta_{3}$ takes a small value, the vibration amplitude is always stable regardless of the value of time delay $\tau$; with the increase of feedback gain $\zeta_{3}$, the vibration amplitude can be unstable for some specific regions of time delay $\tau$; the unstable solutions where a Hopf bifurcation occurs are determined by $G_{1}$. Behaviour is more complicated when $\Omega=1.3$ because three vibration amplitudes exist for some specific regions of time delay $\tau$. The lowest branch is always stable regardless of the value of time delay $\tau$ and remains the same for different feedback gain $\zeta_{3}$ which can be seen in Figure 4 . The vibration amplitude of the lowest branch is smaller compared to the upper two branches. When feedback gain $\zeta_{3}$ takes a small value, the upper branch is stable and the lower branch is unstable which is determined by $G_{2}$; with the increase of feedback gain $\zeta_{3}$, the upper branch can be unstable for some specific regions of time delay $\tau$; the unstable solutions where a Hopf bifurcation occurs are determined by $G_{1}$. 


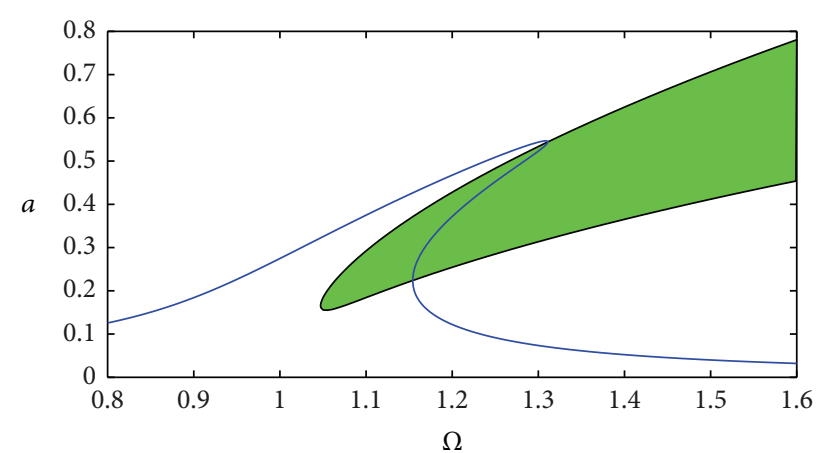

(a) $\tau=\pi / 4$

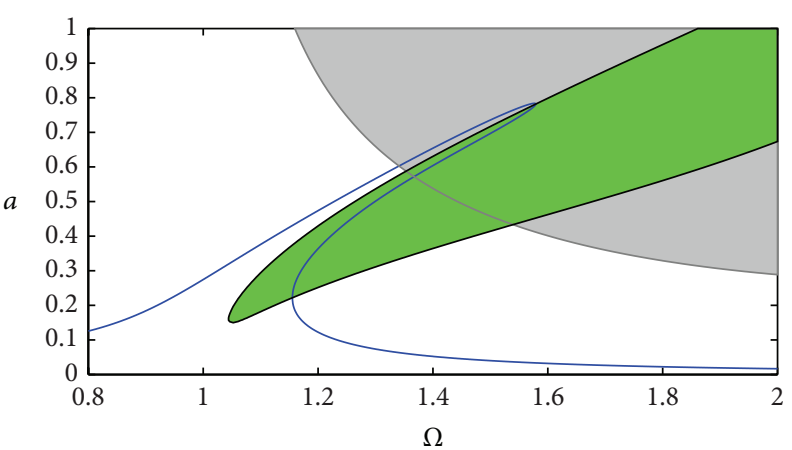

(b) $\tau=\pi / 2$

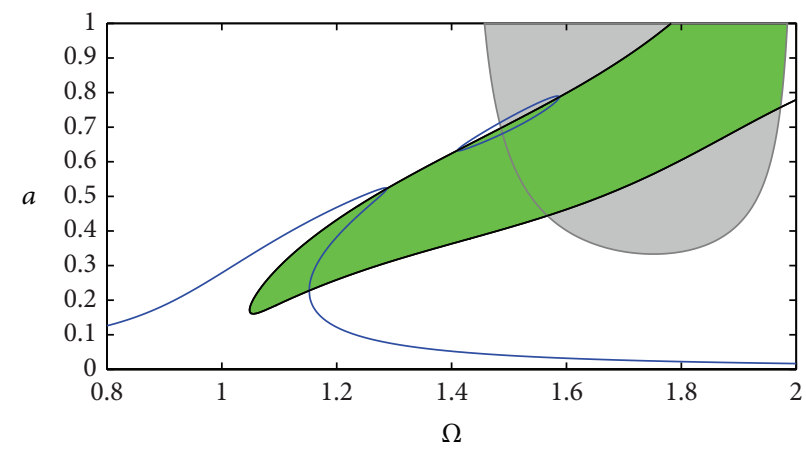

(c) $\tau=7 \pi / 4$

FIGURE 11: Stability boundary of the primary resonance for the controlled system with different time delay $\tau\left(\zeta_{1}=0.025, \zeta_{3}=0.05, f_{1}=0.05\right.$, $k=1$, and $\widehat{\lambda}=0.4$ ).

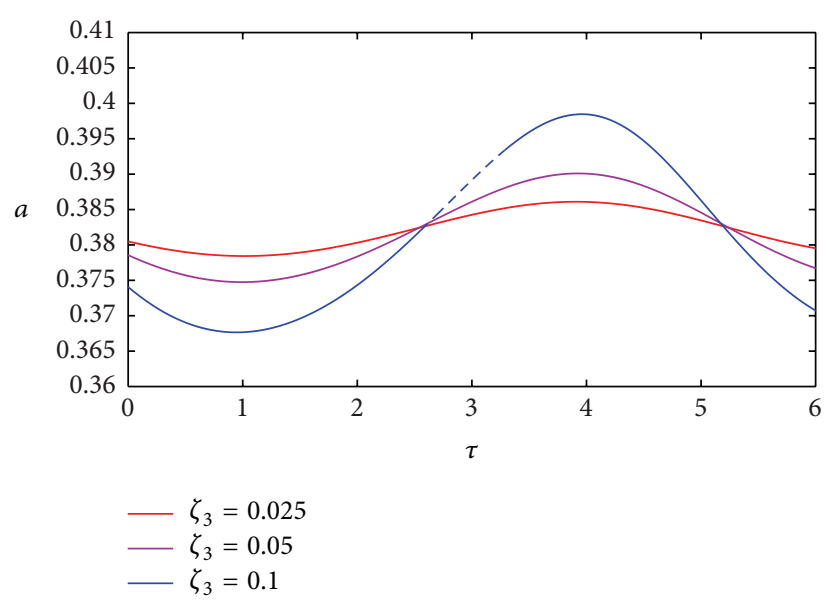

(a) $\Omega=1.1$

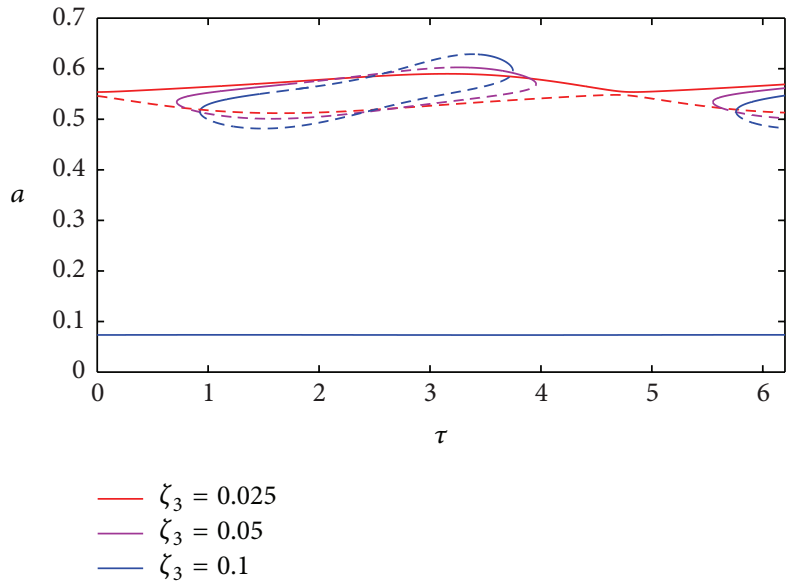

(b) $\Omega=1.3$

FIGURE 12: Effect of the time delay $\tau$ on the stability of vibration amplitude for the controlled system with different feedback gain $\zeta_{3}\left(\zeta_{1}=0.025\right.$, $f_{1}=0.05, k=1$, and $\hat{\lambda}=0.4$ ).

\section{1/3 Subharmonic Resonance}

Since there exist cubic nonlinear terms in the controlled system, when the excitation force amplitude increases to a larger value, 1/3 subharmonic resonance can occur which results in a worse isolation performance. So $1 / 3$ subharmonic resonance of the controlled system is studied in this section.
For the case of $1 / 3$ subharmonic resonance, the excitation force amplitude is larger; it is assumed that

$$
\begin{aligned}
& \zeta_{1}=\varepsilon \widehat{\zeta}_{1}, \\
& \zeta_{3}=\varepsilon \widehat{\zeta}_{3}
\end{aligned}
$$




$$
\begin{gathered}
\beta=\varepsilon \widehat{\beta}, \\
\Omega^{2}=9+\varepsilon \sigma .
\end{gathered}
$$

Then (7) can be rewritten as

$$
\begin{aligned}
\widehat{x}^{\prime \prime}+ & 2 \varepsilon \widehat{\zeta}_{1} \widehat{x}^{\prime}+2 \varepsilon \widehat{\zeta}_{3} \hat{x}^{\prime 3}(T-\tau)+\frac{\left(\Omega^{2}-\varepsilon \sigma\right)}{9} \widehat{x} \\
& +\varepsilon \hat{\beta} \widehat{x}^{3}=f_{1} \cos \Omega T .
\end{aligned}
$$

Substituting (10) and (11) into (33) and equating the coefficients of the same power of $\varepsilon$, the following equations can be obtained:

$$
\begin{gathered}
D_{0}^{2} x_{0}+\frac{\Omega^{2}}{9} x_{0}=f_{1} \cos \Omega T_{0}, \\
D_{0}^{2} x_{1}+\frac{\Omega^{2}}{9} x_{1}=-2 D_{0} D_{1} x_{0}-2 \widehat{\xi}_{1} D_{0} x_{0} \\
-2 \widehat{\xi}_{3}\left[D_{0} x_{0}\left(T_{0}-\tau\right)\right]^{3}-\widehat{\beta} x_{0}^{3}+\frac{\sigma}{9} x_{0} .
\end{gathered}
$$

The solution of (32a) can be written in the form

$$
\begin{gathered}
x_{0}\left(T_{0}, T_{1}\right)=A\left(T_{1}\right) e^{j(\Omega / 3) T_{0}}+\Lambda e^{j \Omega T_{0}}+c c, \\
A\left(T_{1}\right)=\frac{1}{2} a\left(T_{1}\right) e^{j \Phi\left(T_{1}\right)}, \\
\Lambda=-\frac{9 f_{1}}{16 \Omega^{2}} .
\end{gathered}
$$

Substituting (35a) into (34b) and removing secular terms from $(34 b)$ require

$$
\begin{aligned}
& -j \frac{2}{3} \Omega D_{1} A-j \frac{2}{3} \widehat{\xi}_{1} \Omega A-3 \widehat{\beta} A^{2} \bar{A}-3 \widehat{\beta} \bar{A}^{2} \Lambda \\
& \quad-6 \widehat{\beta} A \Lambda^{2}+\frac{1}{9} \sigma A-j \frac{2}{9} \widehat{\zeta}_{3} \Omega^{3} A^{2} \bar{A} e^{-j(\Omega / 3) \tau} \\
& \quad-j 4 \widehat{\zeta}_{3} \Omega^{3} A \Lambda^{2} e^{-j(\Omega / 3) \tau}+j \frac{2}{3} \widehat{\zeta}_{3} \Omega^{3} \bar{A}^{2} \Lambda e^{-j(\Omega / 3) \tau}=0 .
\end{aligned}
$$

Substituting (35b) into (36) and separating the real and imaginary terms, the following equations can be obtained:

$$
\begin{aligned}
a^{\prime}=\frac{1}{\Omega}( & -\Omega \widehat{\zeta}_{1} a-\frac{1}{12} \widehat{\zeta}_{3} \Omega^{3} a^{3} \cos \frac{\Omega}{3} \tau-6 \widehat{\zeta}_{3} \Omega^{3} \Lambda^{2} a \cos \frac{\Omega}{3} \tau \\
& \left.+\frac{9}{4} \widehat{\beta} \Lambda a^{2} \sin 3 \Phi+\frac{1}{2} \widehat{\zeta}_{3} \Omega^{3} \Lambda a^{2} \cos \left(\frac{\Omega}{3} \tau+3 \Phi\right)\right)
\end{aligned}
$$

$$
\begin{aligned}
a \Phi^{\prime}=\frac{1}{\Omega}( & -\frac{1}{6} \sigma a+\frac{9}{8} \widehat{\beta} a^{3}+9 \widehat{\beta} a \Lambda^{2}+\frac{1}{12} \widehat{\zeta}_{3} \Omega^{3} a^{3} \sin \frac{\Omega}{3} \tau \\
& +6 \widehat{\zeta}_{3} \Omega^{3} \Lambda^{2} a \sin \frac{\Omega}{3} \tau+\frac{9}{4} \widehat{\beta} \Lambda a^{2} \cos 3 \Phi \\
& \left.-\frac{1}{2} \widehat{\zeta}_{3} \Omega^{3} \Lambda a^{2} \sin \left(\frac{\Omega}{3} \tau+3 \Phi\right)\right) .
\end{aligned}
$$

Then the amplitude-frequency and phase-frequency relationships of $1 / 3$ subharmonic resonance using the original structural parameter can be given by

$$
\begin{aligned}
\left(\zeta_{1} \Omega\right. & \left.+\frac{1}{12} \zeta_{3} \Omega^{3} a^{2} \cos \frac{\Omega}{3} \tau+6 \zeta_{3} \Omega^{3} \Lambda^{2} \cos \frac{\Omega}{3} \tau\right)^{2} \\
& +\left(\frac{9-\Omega^{2}}{6}+\frac{9}{8} \beta a^{2}+9 \beta \Lambda^{2}+\frac{1}{12} \zeta_{3} \Omega^{3} a^{2} \sin \frac{\Omega}{3} \tau\right. \\
& \left.+6 \zeta_{3} \Omega^{3} \Lambda^{2} \sin \frac{\Omega}{3} \tau\right)^{2} \\
= & \left.\frac{9}{4} \beta \Lambda a-\frac{1}{2} \zeta_{3} \Omega^{3} \Lambda a \sin \frac{\Omega}{3} \tau\right)^{2} \\
& +\left(\frac{1}{2} \zeta_{3} \Omega^{3} \Lambda a \cos \frac{\Omega}{3} \tau\right)^{2}, \\
A_{2}= & \frac{\tan 3 \Phi=\frac{A_{1} C_{1}-A_{2} C_{2}}{A_{1} C_{2}+A_{2} C_{1}}}{6}+\frac{9}{8} \beta a^{2}+9 \beta \Lambda^{2}+\frac{1}{12} \zeta_{3} \Omega^{3} a^{2} \sin \frac{\Omega}{3} \tau \\
A_{1}= & \zeta_{1} \Omega+\frac{1}{12} \zeta_{3} \Omega^{3} a^{2} \cos \frac{\Omega}{3} \tau+6 \zeta_{3} \Omega^{3} \Lambda^{2} \cos \frac{\Omega}{3} \tau \\
& C_{2}=\frac{1}{2} \zeta_{3} \Omega^{3} \Lambda a \sin \frac{\Omega}{3} \tau \\
&
\end{aligned}
$$

Equation (38a) can be written as a polynomial function in $a$ as

$$
\begin{aligned}
& a^{4}-2 p_{3} a^{2}+q_{3}=0 \\
& p_{3}=(\left(-\frac{3}{8} \zeta_{3}^{2} \Omega^{6}-\frac{243}{32} \beta^{2}-\frac{69}{8} \beta \zeta_{3} \Omega^{3} \sin \frac{\Omega}{3} \tau\right) \Lambda^{2} \\
&+\frac{1}{72} \zeta_{3} \Omega^{5} \sin \frac{\Omega}{3} \tau-\frac{1}{12} \zeta_{1} \zeta_{3} \Omega^{4} \cos \frac{\Omega}{3} \tau \\
&\left.-\frac{1}{8} \zeta_{3} \Omega^{3} \sin \frac{\Omega}{3} \tau+\frac{3}{16} \beta \Omega^{2}-\frac{27}{16} \beta\right) \\
&\left(\frac{1}{144} \zeta_{3}^{2} \Omega^{6}+\frac{81}{64} \beta^{2}+\frac{3}{16} \beta \zeta_{3} \Omega^{3} \sin \frac{\Omega}{3} \tau\right)^{-1} \\
& q_{3}=\left(\left(\zeta_{1} \Omega^{2}+6 \zeta_{3} \Omega^{3} \Lambda^{2} \cos \frac{\Omega}{3} \tau\right)^{2}\right. \\
&\left.+\left(\frac{3}{2}+9 \beta \Lambda^{2}+6 \zeta_{3} \Omega^{3} \Lambda^{2} \sin \frac{\Omega}{3} \tau-\frac{\Omega^{2}}{6}\right)^{2}\right) \\
& \cdot\left(\frac{1}{144} \zeta_{3}^{2} \Omega^{6}+\frac{81}{64} \beta^{2}+\frac{3}{16} \beta \zeta_{3} \Omega^{3} \sin \frac{\Omega}{3} \tau\right)^{-1}
\end{aligned}
$$



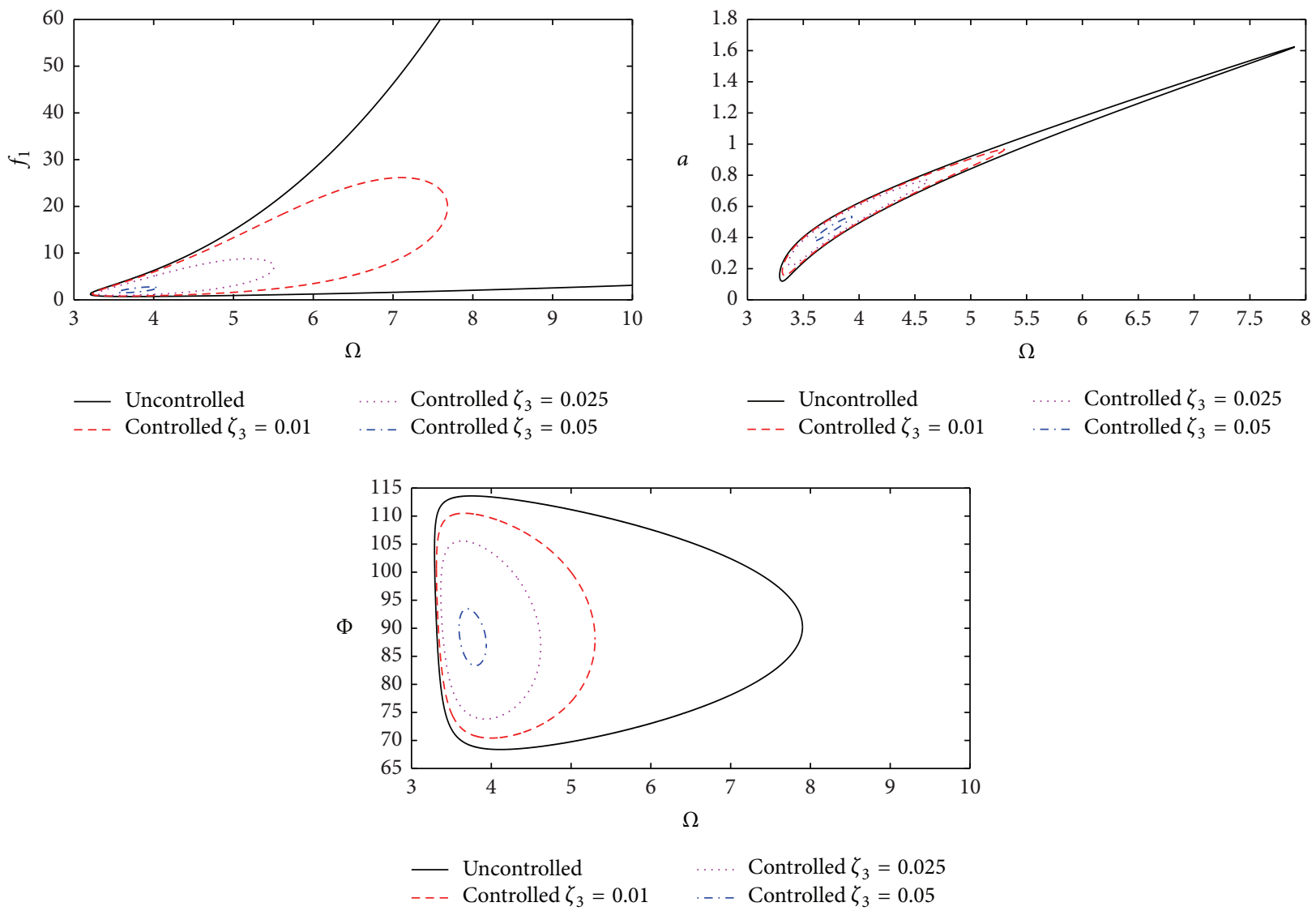

FIGURE 13: Existence regions, amplitude-frequency, and phase-frequency relationships of the 1/3 subharmonic resonance for the controlled system under different feedback gain $\zeta_{3}$ without time delay $\left(\zeta_{1}=0.025, k=1, \hat{\lambda}=0.4\right.$, and $\left.f_{1}=2\right)$.

It can be seen from (39a) that there exists amplitude for a given frequency if $p_{3}>0$ and $p_{3}^{2} \geq q_{3}$ since $q_{3}>0$. Substituting ((39b)-(39c)) into these inequalities, the existence condition of the $1 / 3$ subharmonic resonance can be obtained.

From (38a)-(38f) and (39a)-(39c), it can be seen that the amplitude-frequency, phase-frequency relationships, and existence regions of the $1 / 3$ subharmonic resonance for the controlled system are greatly influenced by the nondimensional feedback gain $\zeta_{3}$ and time delay $\tau$ when the other structural parameters are taking the same values as in the primary resonance part. The effect of the feedback gain $\zeta_{3}$ on the $1 / 3$ subharmonic resonance of the controlled system without time delay can be clearly seen in Figure 13. When the system is uncontrolled, the existence regions and amplitude of the $1 / 3$ subharmonic resonance are relatively large. When the feedback gain $\zeta_{3}$ increases, the existence regions of the $1 / 3$ subharmonic resonance decrease rapidly. So by choosing appreciate value of feedback gain $\zeta_{3}$, the $1 / 3$ subharmonic resonance of the controlled system without time delay can be avoided.

The effect of the time delay $\tau$ on the $1 / 3$ subharmonic resonance of the controlled system can be seen in Figure 14 . Compared to the uncontrolled system, the existence regions of the $1 / 3$ subharmonic resonance for the controlled system with time delay can also become smaller but are larger than the controlled system without time delay. Because when time delay exists, the trigonometric functions appear in both the amplitude-frequency function and existence region function and cannot be eliminated, which can be clearly seen in (37a) and (37b) and (38a)-(38f). So there exist multiple existence regions for the $1 / 3$ subharmonic resonance; the existence regions for larger frequency are not shown in Figure 14 for simplicity. When the time delay exists in the controlled system, the $1 / 3$ subharmonic resonance can occur for a smaller excitation force amplitude and increasing the feedback gain cannot eliminate the $1 / 3$ subharmonic resonance which is undesirable in practical engineering. So in the meaning of controlling $1 / 3$ subharmonic resonance, the controlled system without time delay is better.

The stability analysis of the $1 / 3$ subharmonic resonance for the controlled system can be investigated as the primary resonance case, so it is no longer described here. It can be proven that the upper branch of the $1 / 3$ subharmonic resonance is asymptotically stable, while the lower branch is unstable.

\section{Force Transmissibility}

Force transmissibility is an important index to describe the performance of the vibration isolator. It is defined as the ratio between the force transmitted to the base and the excitation force. In this section, force transmissibility is investigated to evaluate the performance of the controlled HSLDS vibration 

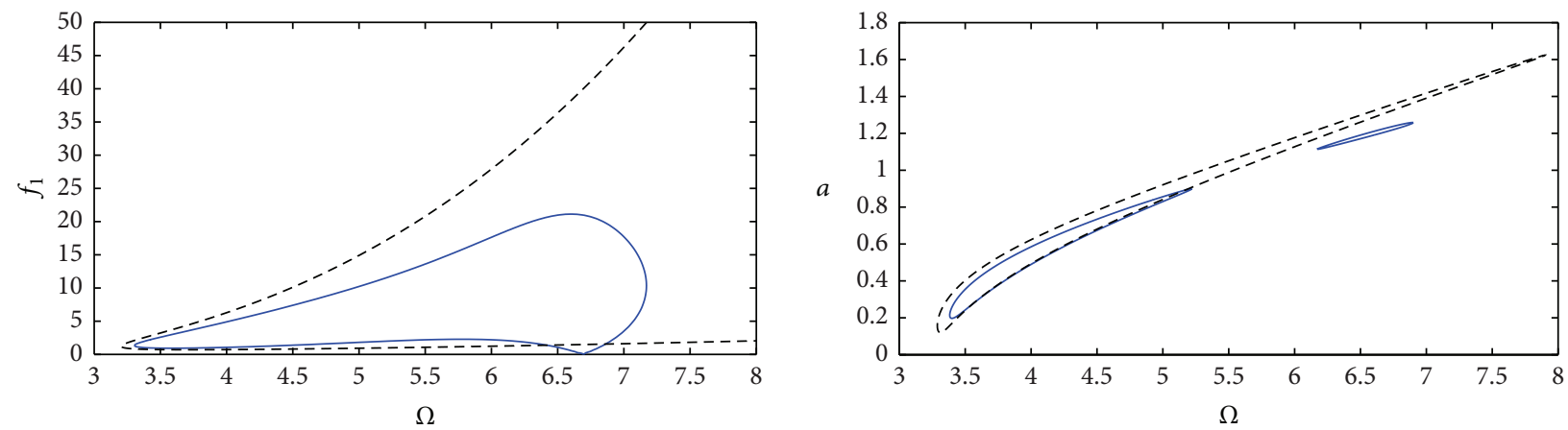

(a) $\tau=\pi / 4$
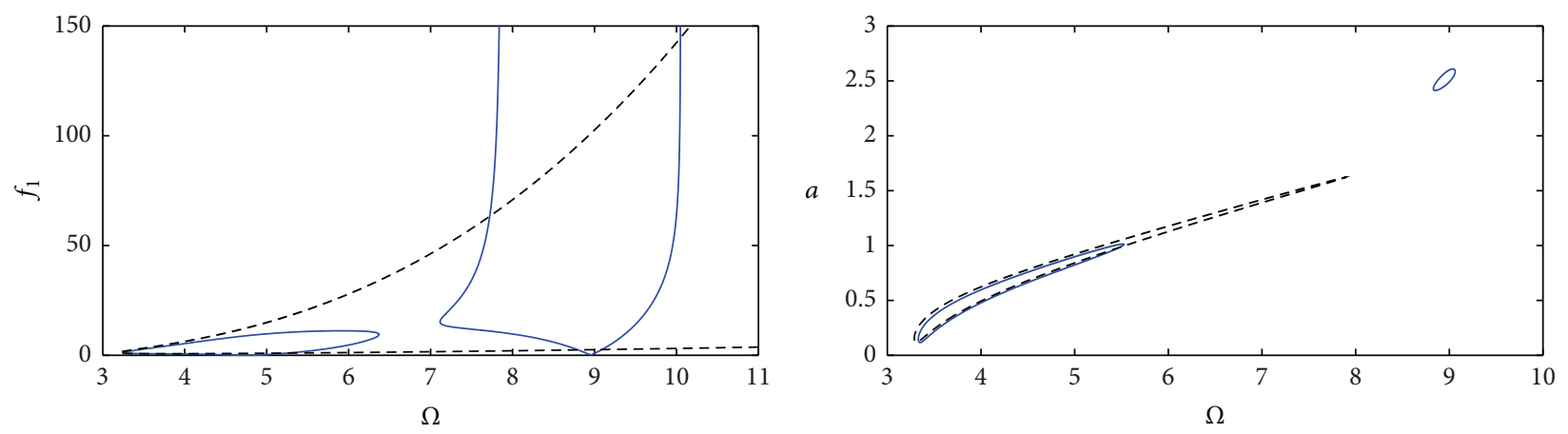

(b) $\tau=2 \pi / 4$
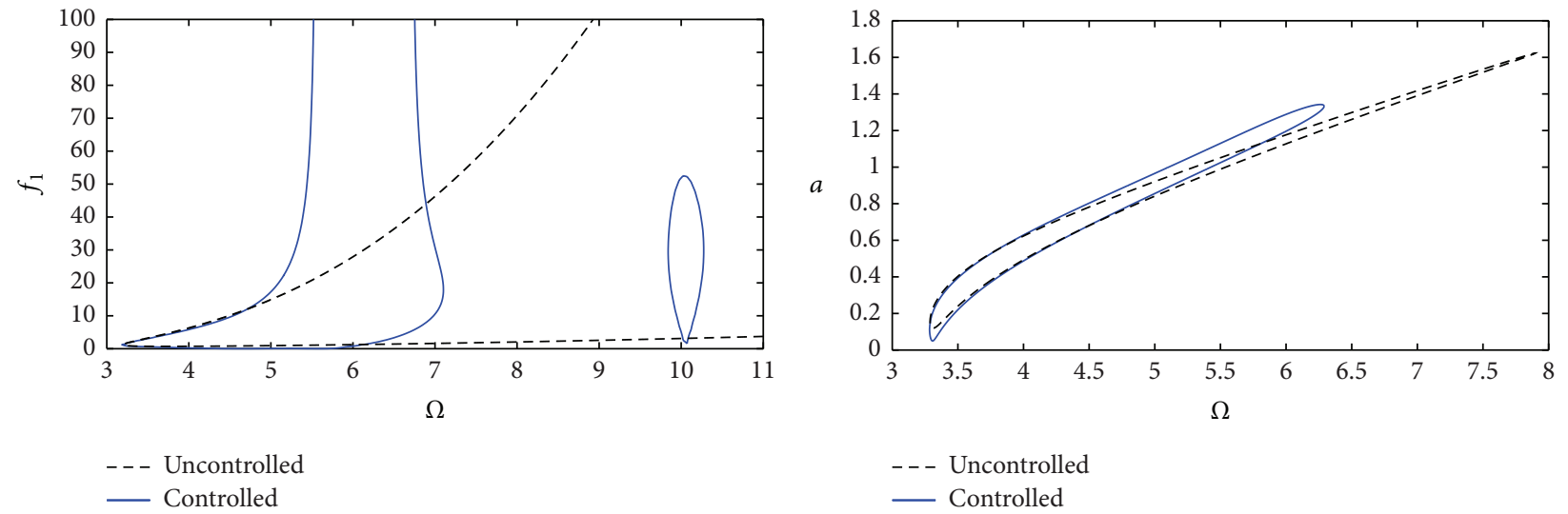

(c) $\tau=3 \pi / 4$

FIGURE 14: Existence regions and amplitude-frequency relationships of the $1 / 3$ subharmonic resonance for the controlled system with different time delay $\tau\left(\zeta_{1}=0.025, \zeta_{3}=0.025, k=1, \hat{\lambda}=0.4\right.$, and $\left.f_{1}=2\right)$.

isolator and compared with an equivalent linear passive vibration isolator. The nondimensional force transmitted to the base through the controlled HSLDS vibration isolator is defined as the following form:

$$
f_{t}=2 \zeta_{1} \widehat{x}^{\prime}+2 \zeta_{3} \hat{x}^{\prime 3}(T-\tau)+\widehat{x}+\widehat{\beta} \widehat{x}^{3} .
$$

Using (13a) and (13b), the nondimensional force is obtained as

$$
\begin{aligned}
f_{t}=( & \left(2 \zeta_{1} \Omega a+\frac{3}{2} \zeta_{3} \Omega^{3} a^{3} \cos \Omega \tau\right)^{2} \\
& \left.+\left(a+\frac{3}{4} \beta a^{3}+\frac{3}{2} \zeta_{3} \Omega^{3} a^{3} \sin \Omega \tau\right)^{2}\right)^{1 / 2} .
\end{aligned}
$$

The force transmissibility of the controlled system can be expressed as

$$
\begin{aligned}
T_{\mathrm{NF}}= & \frac{f_{t}}{f_{1}} \\
= & \left(\left(2 \zeta_{1} \Omega a+\frac{3}{2} \zeta_{3} \Omega^{3} a^{3} \cos \Omega \tau\right)^{2}\right. \\
& \left.+\left(a+\frac{3}{4} \beta a^{3}+\frac{3}{2} \zeta_{3} \Omega^{3} a^{3} \sin \Omega \tau\right)^{2}\right)^{1 / 2} \cdot\left(f_{1}\right)^{-1} .
\end{aligned}
$$




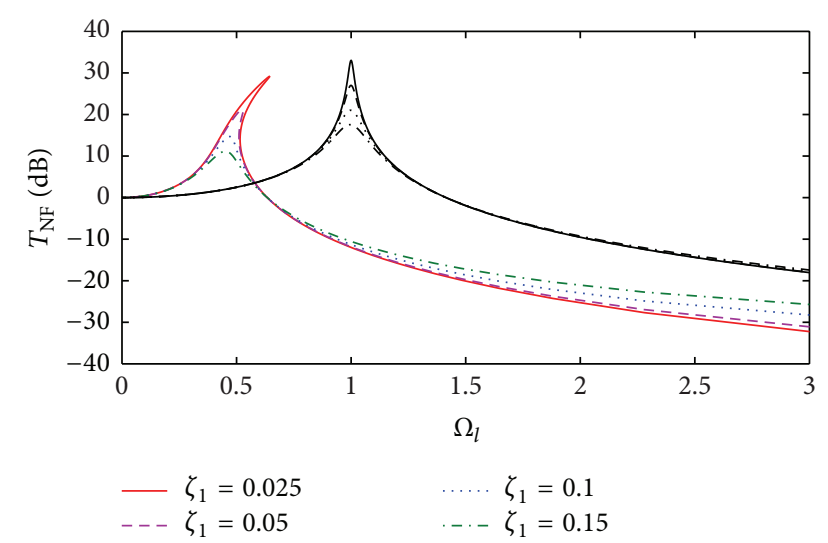

FIGURE 15: Force transmissibility of the uncontrolled system with different damping ratio $\zeta_{1}\left(f_{1}=0.05, k=1\right.$, and $\left.\widehat{\lambda}=0.4\right)$.

It is also of interest to compare the force transmissibility of the controlled system with an equivalent linear one with the same load bearing capacity. The force transmissibility of the equivalent linear vibration isolator is given as

$$
T_{\mathrm{LF}}=\sqrt{\frac{1+4 \zeta_{l}^{2} \Omega_{l}^{2}}{\left(1-\Omega_{l}^{2}\right)^{2}+4 \zeta_{l}^{2} \Omega_{l}^{2}}}
$$

where $\xi_{l}, \Omega_{l}$ are the damping ratio and frequency ratio of the equivalent linear vibration isolator, respectively. Since the HSLDS vibration isolator is comprised of load bearing element and stiffness correctors, the equivalent linear one is the HSLDS vibration isolator with the stiffness correctors removed; then $\xi_{l}$ and $\Omega_{l}$ should satisfy the following conditions:

$$
\begin{gathered}
\zeta_{l}=\sqrt{\alpha} \zeta_{1}, \\
\Omega_{l}=\frac{\Omega}{\sqrt{\alpha}} .
\end{gathered}
$$

For the sake of consistency, when plotting the force transmissibility of the controlled system and an equivalent linear one on the same figure, the values on the frequency axis have to comply with (44b). The force transmissibility of these two vibration isolators is expressed in $\mathrm{dB}$ scale as $20 \log 10\left(T_{\mathrm{NF}}\right)$ and $20 \log 10\left(T_{\mathrm{LF}}\right)$, respectively. As can be seen in (42), the force transmissibility of the controlled system is greatly influenced by the nondimensional damping ratio $\zeta_{1}$, feedback gain $\zeta_{3}$, and time delay $\tau$ when the other structural parameters are chosen. The following discussion investigates the effects of these parameters on the force transmissibility.

Force transmissibility of the uncontrolled system with different damping ratio $\zeta_{1}$ is shown in Figure 15; the force transmissibility of the equivalent linear one is also plotted in the same figure for comparison. From Figure 15, it can be seen that when the damping ratio is smaller, the jump phenomenon occurs and the smaller the damping ratio is, the larger the force transmissibility curve bends to the right; then the HSLDS vibration isolator can lead to an undesirable

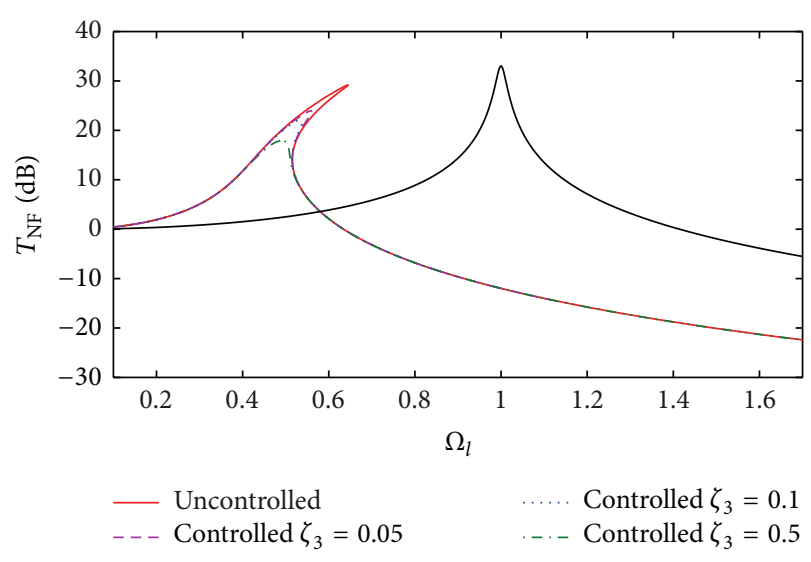

FIGURE 16: Force transmissibility of the controlled system under different feedback gain $\zeta_{3}$ without time delay $\left(\zeta_{1}=0.025, f_{1}=0.05\right.$, $k=1$, and $\hat{\lambda}=0.4$ ).

isolation performance compared to the linear one. When the damping ratio increases, it can significantly reduce the vibration amplitude around the resonance frequency region and the isolation frequency band is larger than the linear one; then the performance of the HSLDS vibration isolator is superior to the linear one, but it results in a poor performance in the high frequency band where the isolation is required.

Force transmissibility of the controlled system under different feedback gain $\zeta_{3}$ without time delay is shown in Figure 16. The effect of feedback gain $\zeta_{3}$ on the force transmissibility can be clearly seen in this figure. When the feedback gain $\zeta_{3}$ increases, the vibration amplitude around the resonance frequency region decreases; the jump phenomenon can be eliminated and can achieve a better isolation performance in the high frequency band where the isolation is required. Thus the cubic velocity feedback control strategy can solve the dilemma existing in the passive vibration isolators with linear damping.

Force transmissibility of the controlled system with different time delay $\tau$ is shown in Figure 17. The effect of time delay $\tau$ on the force transmissibility can also be interpreted by the equivalent damping ratio and equivalent resonance frequency. Then the force transmissibility of the controlled system can be obtained as

$$
T_{\mathrm{NF}}=\frac{f_{t}}{f_{1}}=\frac{\left(\sqrt{\left(2 \zeta_{\mathrm{eq}} \Omega a\right)^{2}+\left(a w_{\mathrm{eq}}^{2}\right)^{2}}\right)}{f_{1}}
$$

The time delay $\tau$ can influence the peak amplitude and resonance frequency of the force transmissibility just as the primary resonance case which can achieve a better or poorer isolation performance than the uncontrolled system, so it is no longer described in detail here. Another important feature that should be addressed is that the time delay $\tau$ does not influence the isolation performance in the high frequency band where the isolation is required. 


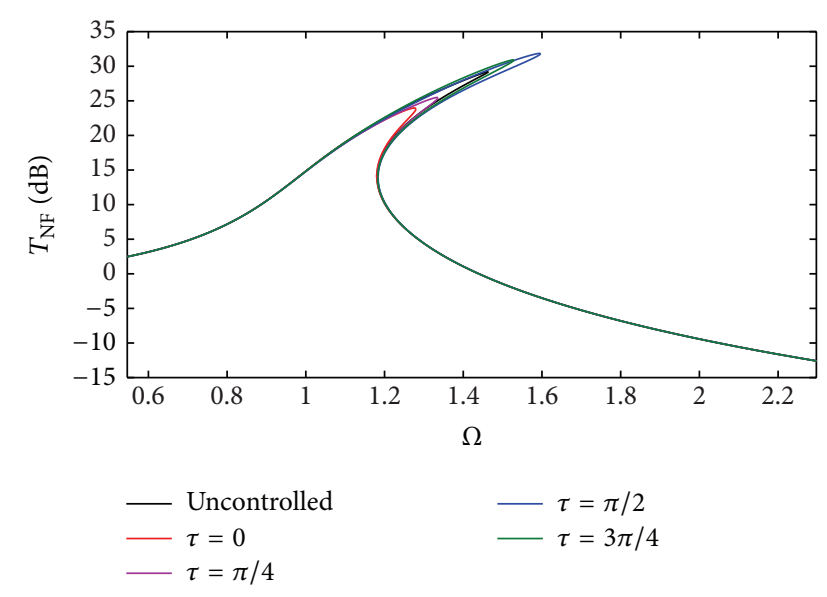

FIGURE 17: Force transmissibility of the controlled system with different time delay $\tau\left(\zeta_{1}=0.025, \zeta_{3}=0.025, f_{1}=0.05, k=1\right.$, and $\hat{\lambda}=0.4$ ).

\section{Conclusion}

This paper proposes the time-delayed cubic velocity feedback control strategy to improve the isolation performance of HSLDS vibration isolator. Both the resonance and performance of the controlled HSLDS vibration isolator are considered. The primary resonance of the controlled system is obtained by using the multiple scales method. The equivalent damping ratio and equivalent resonance frequency are defined to study the effects of feedback gain $\zeta_{3}$ and time delay $\tau$ on the primary resonance. When time delay $\tau=0$, with the increase of feedback gain $\zeta_{3}$, the equivalent damping ratio becomes larger; the peak amplitude of the primary resonance decreases and is smaller than the uncontrolled system. When time delay $\tau$ exists in the controlled system, the equivalent damping ratio and equivalent resonance frequency can be considered as the periodic functions of time delay $\tau$; the peak amplitude of the primary resonance can be smaller or larger than the uncontrolled system based on the value of time delay $\tau$. A closed-loop response curve is found above the primary resonance because multiple solutions exist in these frequency bands when time delay $\tau$ takes some special values.

The jump phenomenon analysis of the controlled system is then investigated. When time delay $\tau=0$, it is convenient to use the method of Sylvester resultant to determine the critical surface of the structural parameters which lead to no jump phenomenon and the jump frequencies when jump phenomenon occurs. When time delay $\tau$ exists in the controlled system, the method of Sylvester resultant cannot be used because the trigonometric functions exist in the amplitude-frequency function of the primary resonance; the critical boundary which leads to no jump phenomenon and jump frequencies can be obtained by using the optimization method but is more complicated. The stability analysis of the controlled system is also considered; it is found that the stability boundary of the primary resonance is greatly influenced by the feedback gain $\zeta_{3}$ and time delay $\tau$.

The $1 / 3$ subharmonic resonance of the controlled system is studied by using the multiple scales method. When time delay $\tau=0$, with the increase of feedback gain $\zeta_{3}$, the existence regions of the $1 / 3$ subharmonic resonance decrease rapidly and the $1 / 3$ subharmonic resonance can be avoided by choosing appreciate value of feedback gain $\zeta_{3}$. When time delay $\tau$ exists in the controlled system, the $1 / 3$ subharmonic resonance can occur for a smaller excitation force amplitude and increasing the feedback gain cannot eliminate the $1 / 3$ subharmonic resonance. So in the meaning of controlling $1 / 3$ subharmonic resonance, the controlled system without time delay is better.

Last, the force transmissibility of the controlled system is considered. When the feedback gain $\zeta_{3}$ increases, the vibration amplitude around the resonance frequency region decreases and the isolation frequency band is larger which is superior to the equivalent linear passive vibration isolator. It can also achieve a better isolation performance in the high frequency band where the isolation is required. Thus the cubic velocity feedback control strategy can solve the dilemma existing in the passive vibration isolators with linear damping.

\section{Appendix}

Consider

$$
\begin{aligned}
b_{0}= & -\frac{256}{27} \zeta_{1}^{2} \zeta_{3}^{2} \beta^{2}-\frac{4096}{27} \zeta_{1}^{5} \zeta_{3}^{3} \beta+\frac{1024}{9} \zeta_{1}^{3} \zeta_{3}^{3} \beta-\frac{1024}{27} \zeta_{1}^{2} \zeta_{3}^{4}, \\
b_{2}= & -\frac{32}{3} \zeta_{1}^{2} \zeta_{3}^{2} \beta^{3}+\left(\frac{1024}{9} \zeta_{1}^{2}-\frac{512}{3} \zeta_{1}^{4}-\frac{64}{3}\right) \zeta_{1} \zeta_{3}^{3} \beta^{2} \\
& +\left(-\frac{2048}{9} \zeta_{1}^{4}-\frac{512}{3} \zeta_{1}^{2}+\frac{640}{3}\right) \zeta_{1}^{2} \zeta_{3}^{4} \beta \\
& -\left(\frac{512}{9} \zeta_{1}^{2}+\frac{256}{3}\right) \zeta_{1} \zeta_{3}^{5}, \\
b_{4}= & \zeta_{3}^{2} \beta^{4}-64 \zeta_{1} \zeta_{3}^{3} \beta^{3}+\left(-256 \zeta_{1}^{6}-640 \zeta_{1}^{4}+624 \zeta_{1}^{2}-8\right) \zeta_{3}^{4} \beta^{2} \\
& -\left(64+256 \zeta_{1}^{2}\right) \zeta_{1} \zeta_{3}^{5} \beta-48 \zeta_{3}^{6}, \\
b_{6}= & -30 \zeta_{1} \zeta_{3}^{3} \beta^{4}+\left(-480 \zeta_{1}^{4}+360 \zeta_{1}^{2}-54\right) \zeta_{3}^{4} \beta^{3} \\
& +\left(-864 \zeta_{1}^{2}+528\right) \zeta_{1} \zeta_{3}^{5} \beta^{2}-216 \zeta_{3}^{6} \beta, \\
b_{8}= & -81 \zeta_{3}^{4} \beta^{4}+\left(-1296 \zeta_{1}^{2}+972\right) \zeta_{1} \zeta_{3}^{5} \beta^{3}-324 \zeta_{3}^{6} \beta^{2}, \\
b_{10}= & -\frac{81}{2} \zeta_{3}^{4} \beta^{5}+\left(-648 \zeta_{1}^{2}+486\right) \zeta_{1} \zeta_{3}^{5} \beta^{4}-162 \zeta_{3}^{6} \beta^{3}, \\
b_{1}= & b_{3}=b_{5}=b_{7}=b_{9}=0, \\
c_{0}= & f_{1}^{4} \\
c_{2}= & 27 \zeta_{1} \zeta_{3} f_{1}^{4}, \\
c_{4}= & \left(135 \zeta_{1}^{2}+\frac{81}{2}\right) \zeta_{3}^{2} f_{1}^{4} \\
& +\left(96 \zeta_{3} \zeta_{1}^{4}-96 \zeta_{3} \zeta_{1}^{2}+6 \beta \zeta_{1}+12 \zeta_{3}\right) \zeta_{1} f_{1}^{2},
\end{aligned}
$$




$$
\begin{aligned}
& c_{6}=-\frac{2187}{32} \zeta_{3}^{4} f_{1}^{6}+\left(-54 \zeta_{3} \zeta_{1}^{3}+243 \zeta_{3} \zeta_{1}+\frac{729}{16} \beta\right) \zeta_{3}^{2} f_{1}^{4} \\
& +\left(\left(144 \zeta_{1}^{4}-270 \zeta_{1}^{2}+9\right) \zeta_{3}^{2}\right. \\
& \left.+\left(-54 \beta \zeta_{1}^{3}+72 \beta \zeta_{1}\right) \zeta_{3}-\frac{9}{4} \beta^{2}\right) f_{1}^{2}, \\
& c_{8}=\left(\frac{2187}{16} \zeta_{3}+\frac{729}{4} \zeta_{1} \beta\right) \zeta_{3}^{3} f_{1}^{4} \\
& +\left(\left(54 \zeta_{1}^{3}-243 \zeta_{1}\right) \zeta_{3}^{2}\right. \\
& \left.+\left(108 \beta \zeta_{1}^{4}-189 \beta \zeta_{1}^{2}+\frac{567}{8} \beta\right) \zeta_{3}+27 \zeta_{1} \beta^{2}\right) \zeta_{3} f_{1}^{2} \\
& +144 \beta \zeta_{3} \zeta_{1}^{5}-108 \beta \zeta_{3} \zeta_{1}^{3}+\left(9 \beta^{2}+36 \zeta_{3}^{2}\right) \zeta_{1}^{2}, \\
& c_{10}=\frac{6561}{64} \beta \zeta_{3}^{4} f_{1}^{4}+\left(-\frac{729}{8} \zeta_{3}^{2}-\frac{1701}{8} \beta \zeta_{1} \zeta_{3}+\frac{1215}{16} \beta^{2}\right) \zeta_{3}^{2} f_{1}^{2} \\
& +54 \zeta_{1} \zeta_{3}^{3}+\left(216 \zeta_{1}^{2}-135\right) \beta \zeta_{1}^{2} \zeta_{3}^{2} \\
& +\left(108 \zeta_{1}^{4}-81 \zeta_{1}^{2}+\frac{27}{2}\right) \beta^{2} \zeta_{1} \zeta_{3}+\frac{27}{4} \beta^{3} \zeta_{1}^{2} \\
& c_{12}=\left(-\frac{2187}{16} \zeta_{3}^{2}+\left(-\frac{243}{8} \zeta_{1}^{2}+\frac{729}{16}\right) \beta \zeta_{1} \zeta_{3}+\frac{3159}{128} \beta^{2}\right) \beta \zeta_{3}^{2} f_{1}^{2} \\
& +\frac{81}{4} \zeta_{3}^{4}+\left(81 \zeta_{1}^{2}+\frac{81}{4}\right) \beta \zeta_{1} \zeta_{3}^{3} \\
& +\left(324 \zeta_{1}^{4}-243 \zeta_{1}^{2}+\frac{81}{16}\right) \beta^{2} \zeta_{3}^{2}+\frac{81}{4} \beta^{3} \zeta_{1} \zeta_{3} \\
& c_{14}=\left(-\zeta_{3}+\beta \zeta_{1}\right) \frac{6561}{128} \beta^{2} \zeta_{3}^{3} f_{1}^{2}+\frac{729}{16} \beta \zeta_{3}^{4} \\
& +\frac{243}{32} \beta^{4} \zeta_{1} \zeta_{3}+\left(\frac{729}{4} \zeta_{1}^{2}-\frac{1701}{16}\right) \beta^{2} \zeta_{1} \zeta_{3}^{3} \\
& +\left(\frac{243}{2} \zeta_{1}^{4}-\frac{729}{8} \zeta_{1}^{2}+\frac{729}{64}\right) \beta^{3} \zeta_{3}^{2} \\
& c_{16}=\frac{2187}{64} \beta^{2} \zeta_{3}^{4}+\left(\frac{2187}{16} \zeta_{1}^{2}-\frac{6561}{64}\right) \beta^{3} \zeta_{1} \zeta_{3}^{3}+\frac{2187}{256} \beta^{4} \zeta_{3}^{2}, \\
& c_{18}=\frac{2187}{256} \beta^{3} \zeta_{3}^{4}+\left(\frac{2187}{64} \zeta_{1}^{2}-\frac{6561}{256}\right) \beta^{4} \zeta_{1} \zeta_{3}^{3}+\frac{2187}{1024} \beta^{5} \zeta_{3}^{2}, \\
& c_{1}=c_{3}=c_{5}=c_{7}=c_{9}=c_{11}=c_{13}=c_{15}=c_{17}=0 \text {. }
\end{aligned}
$$

\section{Conflict of Interests}

The authors declare that there is no conflict of interests regarding the publication of this paper.

\section{Acknowledgments}

This work was supported by Funding of Jiangsu Innovation Program for Graduate Education (Grant no. KYLX_0243) and the Fundamental Research Funds for the Central Universities. It is also supported by the basic research of Nanjing University of Aeronautics and Astronautics Business Inte-gration of Science and Engineering Project funds (NZ2015103).

\section{References}

[1] P. Alabuzhev, A. Gritchin, L. Kim, G. Migirenko, V. Chon, and P. Stepanov, Vibration Protecting and Measuring Systems with Quasi-Zero Stiffness, Hemisphere Publishing, New York, NY, USA, 1989.

[2] R. A. Ibrahim, "Recent advances in nonlinear passive vibration isolators," Journal of Sound and Vibration, vol. 314, no. 3-5, pp. 371-452, 2008.

[3] A. Carrella, M. J. Brennan, and T. P. Waters, "Static analysis of a passive vibration isolator with quasi-zero-stiffness characteristic," Journal of Sound and Vibration, vol. 301, no. 3-5, pp. 678689, 2007.

[4] I. Kovacic, M. J. Brennan, and T. P. Waters, "A study of a nonlinear vibration isolator with a quasi-zero stiffness characteristic," Journal of Sound and Vibration, vol. 315, no. 3, pp. 700-711, 2008.

[5] A. Carrella, M. J. Brennan, T. P. Waters, and V. Lopes Jr., "Force and displacement transmissibility of a nonlinear isolator with high-static-low-dynamic-stiffness," International Journal of Mechanical Sciences, vol. 55, no. 1, pp. 22-29, 2012.

[6] Y. Wang, S. M. Li, J. Y. Li, X. X. Jiang, and C. Cheng, "Response and performance of a nonlinear vibration isolator with highstatic-low-dynamic-stiffness under shock excitations," Journal of Vibroengineering, vol. 16, no. 7, pp. 3382-3398, 2014.

[7] T. D. Le and K. K. Ahn, "A vibration isolation system in low frequency excitation region using negative stiffness structure for vehicle seat," Journal of Sound and Vibration, vol. 330, no. 26, pp. 6311-6335, 2011.

[8] W. S. Robertson, M. R. F. Kidner, B. S. Cazzolato, and A. C. Zander, "Theoretical design parameters for a quasi-zero stiffness magnetic spring for vibration isolation," Journal of Sound and Vibration, vol. 326, no. 1-2, pp. 88-103, 2009.

[9] N. Zhou and K. Liu, "A tunable high-static-low-dynamic stiffness vibration isolator," Journal of Sound and Vibration, vol. 329, no. 9, pp. 1254-1273, 2010.

[10] D. L. Xu, Q. P. Yu, J. X. Zhou, and S. R. Bishop, "Theoretical and experimental analyses of a nonlinear magnetic vibration isolator with quasi-zero-stiffness characteristic," Journal of Sound and Vibration, vol. 332, no. 14, pp. 3377-3389, 2013.

[11] K. Shin, "Experimental investigation of the vibration transmissibility of a magnet-spring vibration isolator under random excitation," Journal of Vibroengineering, vol. 16, no. 4, pp. 17451752, 2014.

[12] X. Liu, X. Huang, and H. Hua, "On the characteristics of a quasizero stiffness isolator using Euler buckled beam as negative stiffness corrector," Journal of Sound and Vibration, vol. 332, no. 14, pp. 3359-3376, 2013.

[13] X. C. Huang, X. T. Liu, J. Y. Sun, Z. Y. Zhang, and H. X. Hua, "Effect of the system imperfections on the dynamic response of a high-static-low-dynamic stiffness vibration isolator," Nonlinear Dynamics, vol. 76, no. 2, pp. 1157-1167, 2014.

[14] A. D. Shaw, S. A. Neild, and D. J. Wagg, "Dynamic analysis of high static low dynamic stiffness vibration isolation mounts," Journal of Sound and Vibration, vol. 332, no. 6, pp. 1437-1455, 2013. 
[15] A. D. Shaw, S. A. Neild, D. J. Wagg, P. M. Weaver, and A. Carrella, "A nonlinear spring mechanism incorporating a bistable composite plate for vibration isolation," Journal of Sound and Vibration, vol. 332, no. 24, pp. 6265-6275, 2013.

[16] H. Y. Hu, E. H. Dowell, and L. N. Virgin, "Resonances of a harmonically forced Duffing oscillator with time delay state feedback," Nonlinear Dynamics, vol. 15, no. 4, pp. 311-327, 1998.

[17] J. C. Ji, "Local bifurcation control of a forced single-degree-offreedom nonlinear system: saddle-node bifurcation," Nonlinear Dynamics, vol. 25, no. 4, pp. 369-382, 2001.

[18] J. C. Ji and A. Y. T. Leung, "Resonances of a non-linear s.d.o.f. system with two time-delays in linear feedback control," Journal of Sound and Vibration, vol. 253, no. 5, pp. 985-1000, 2002.

[19] Y. J. Shen and M. Ahmadian, "Nonlinear dynamical analysis on four semi-active dynamic vibration absorbers with time delay," Shock and Vibration, vol. 20, no. 4, pp. 649-663, 2013.

[20] R. Rusinek, A. Weremczuk, K. Kecik, and J. Warminski, "Dynamics of a time delayed duffing oscillator," International Journal of Non-Linear Mechanics, vol. 65, pp. 98-106, 2014.

[21] X. Sun, J. Xu, X. Jing, and L. Cheng, "Beneficial performance of a quasi-zero-stiffness vibration isolator with time-delayed active control," International Journal of Mechanical Sciences, vol. 82, no. 1, pp. 32-40, 2014.

[22] A. H. Nayfeh and D. T. Mook, Nonlinear Oscillations, Wiley, New York, NY, USA, 1979.

[23] A. Narimani, M. F. Golnaraghi, and G. N. Jazar, "Sensitivity analysis of the frequency response of a piecewise linear system in a frequency island," Journal of Vibration and Control, vol. 10, no. 2, pp. 175-198, 2004.

[24] P. Malatkar and A. H. Nayfeh, "Calculation of the jump frequencies in the response of s.d.o.f. non-linear systems," Journal of Sound and Vibration, vol. 254, no. 5, pp. 1005-1011, 2002.

[25] C. E. Wee and R. N. Goldman, "Elimination and resultantspart 1: elimination and bivariate resultants," IEEE Computer Graphics and Applications, vol. 15, no. 1, pp. 69-77, 1995.

[26] L. W. Griffiths, Introduction to the Theory of Equations, Wiley, New York, NY, USA, 1947.

[27] G. N. Jazar, R. Houim, A. Narimani, and M. F. Golnaraghi, "Frequency response and jump avoidance in a nonlinear passive engine mount," Journal of Vibration and Control, vol. 12, no. 11, pp. 1205-1237, 2006. 

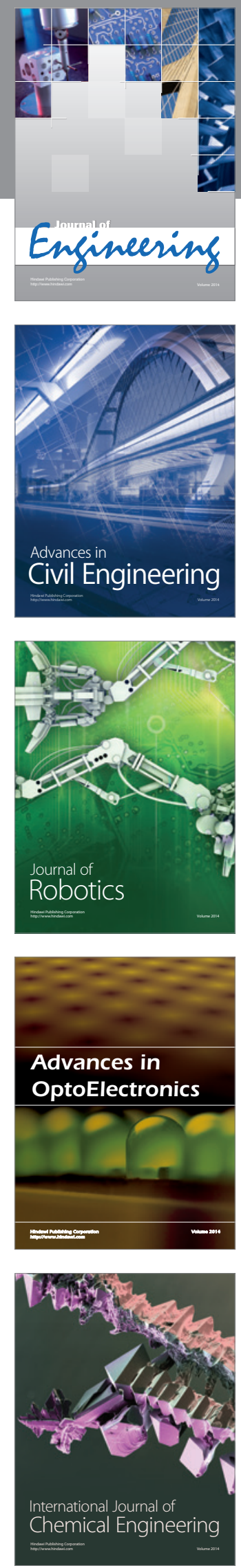

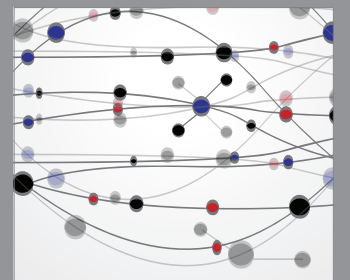

The Scientific World Journal
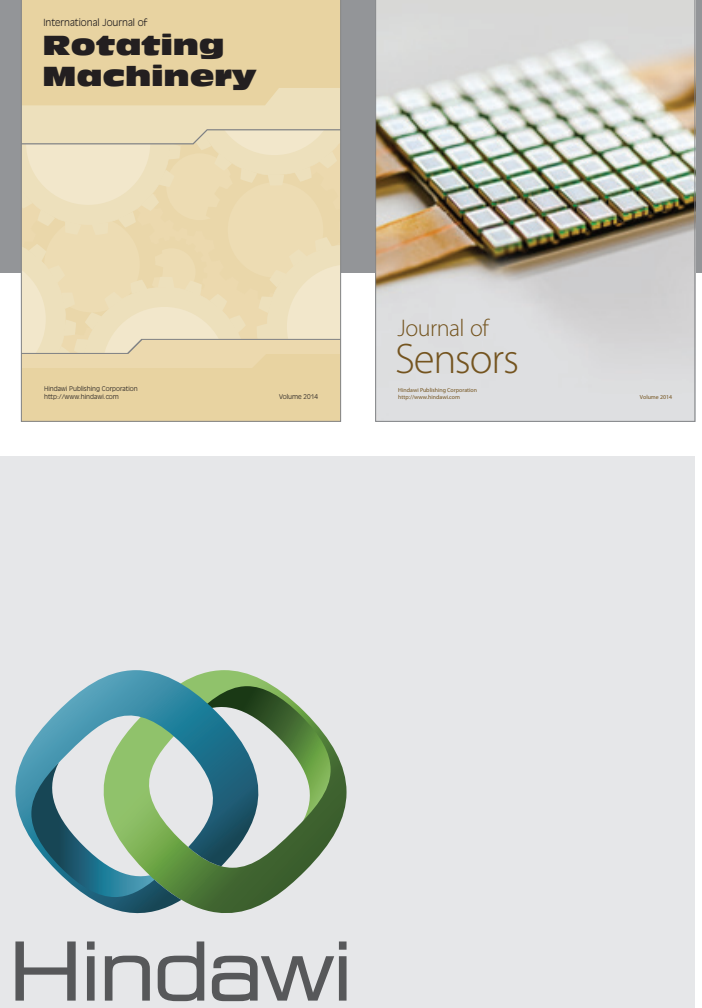

Submit your manuscripts at http://www.hindawi.com
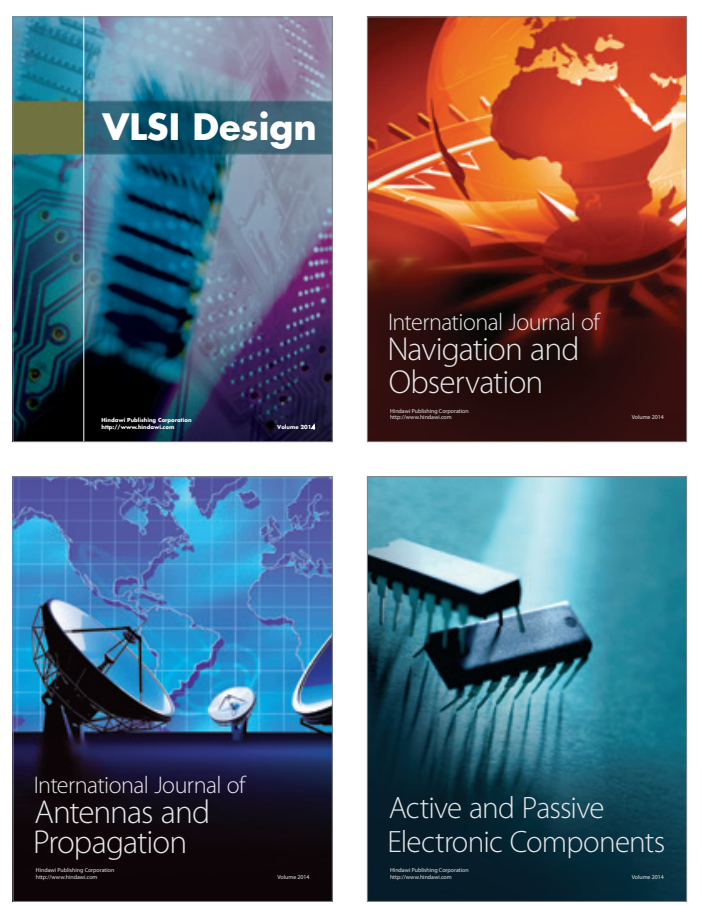
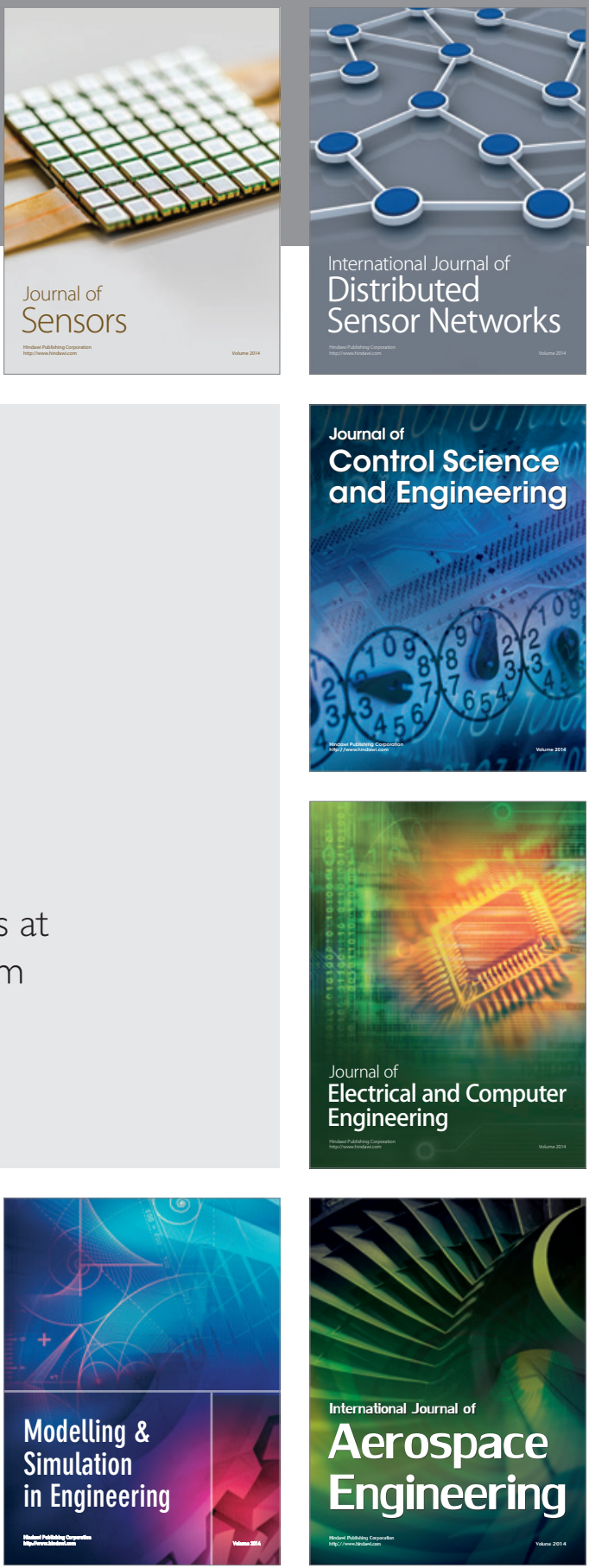

Journal of

Control Science

and Engineering
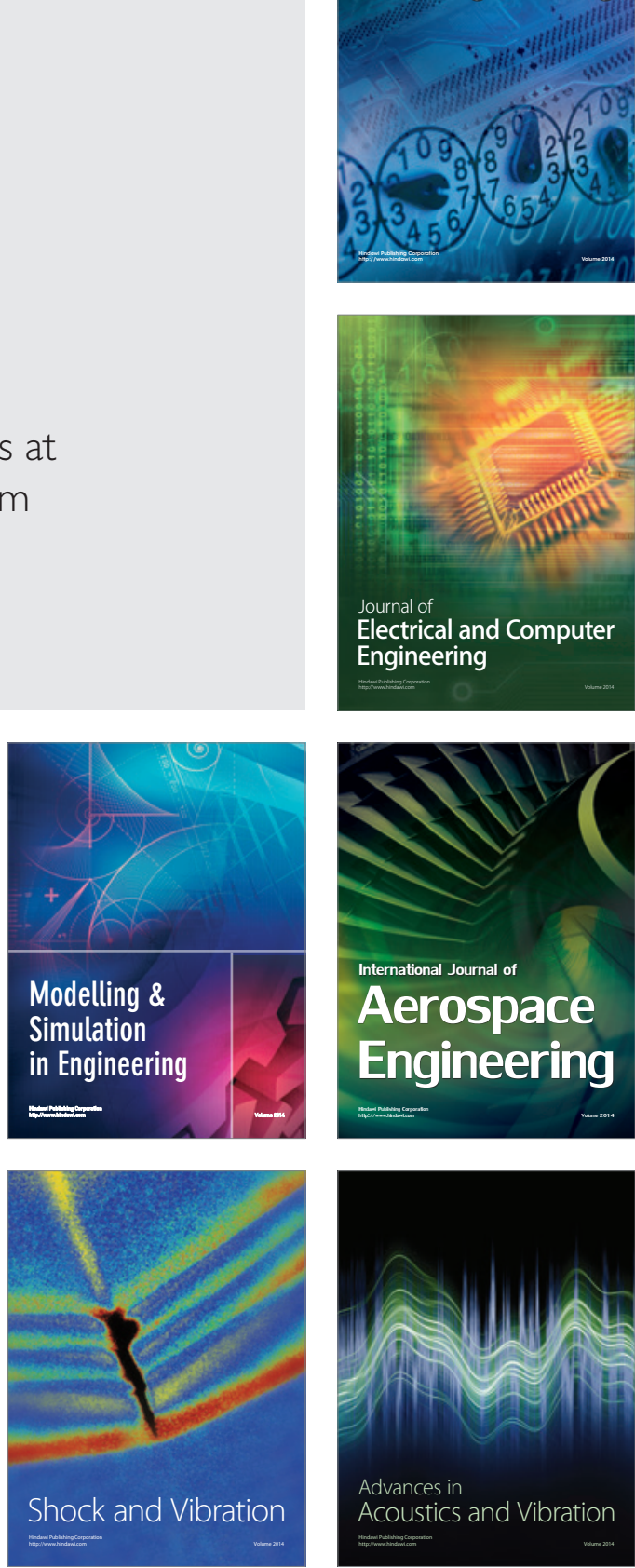